\title{
Search for Neutral Heavy Leptons Produced in Z Decays
}

\author{
DELPHI Collaboration
}

\begin{abstract}
Weak isosinglet Neutral Heavy Leptons $\left(\nu_{m}\right)$ have been searched for using data collected by the DELPHI detector corresponding to $3.3 \times 10^{6}$ hadronic $Z^{0}$ decays at LEP1. Four separate searches have been performed, for short-lived $\nu_{m}$ production giving monojet or acollinear jet topologies, and for long-lived $\nu_{m}$ giving detectable secondary vertices or calorimeter clusters. No indication of the existence of these particles has been found, leading to an upper limit for the branching ratio $B R\left(Z^{0} \rightarrow \nu_{m} \bar{\nu}\right)$ of about $1.3 \times 10^{-6}$ at $95 \%$ confidence level for $\nu_{m}$ masses between 3.5 and $50 \mathrm{GeV} / c^{2}$. Outside this range the limit weakens rapidly with the $\nu_{m}$ mass. The results are also interpreted in terms of limits for the single production of excited neutrinos.
\end{abstract}


P.Abreu ${ }^{21}$, W.Adam ${ }^{50}$, T.Adye ${ }^{37}$, I.Ajinenko ${ }^{42}$, G.D.Alekseev ${ }^{16}$, R.Alemany ${ }^{49}$, P.P.Allport ${ }^{22}$, S.Almehed ${ }^{24}$, U.Amaldi $^{9}$, S.Amato ${ }^{47}$, A.Andreazza ${ }^{28}$, M.L.Andrieux ${ }^{14}$, P.Antilogus ${ }^{9}$, W-D.Apel ${ }^{17}, \quad$ B.Asman ${ }^{44}$, J-E.Augustin ${ }^{25}$, A.Augustinus ${ }^{9}$, P.Baillon ${ }^{9}$, P.Bambade ${ }^{19}$, F.Barao $^{21}$, M.Barbi $^{47}$, G.Barbiellini ${ }^{46}$, D.Y.Bardin ${ }^{16}$, G.Barker $^{9}$, A.Baroncelli ${ }^{40}$, O.Barring ${ }^{24}$, J.A.Barrio ${ }^{26}$, W.Bartl ${ }^{50}$, M.J.Bates ${ }^{37}$, M.Battaglia ${ }^{15}$, M.Baubillier ${ }^{23}$, J.Baudot $^{39}$, K-H.Becks ${ }^{52}$, M.Begalli ${ }^{6}$, P.Beilliere ${ }^{8}$, Yu.Belokopytov ${ }^{9,53}$, A.C.Benvenuti ${ }^{5}$, M.Berggren ${ }^{47}$, D.Bertini $^{25}$, D.Bertrand ${ }^{2}$, M.Besancon ${ }^{39}$, F.Bianchi ${ }^{45}$, M.Bigi ${ }^{45}$, M.S.Bilenky ${ }^{16}$, P.Billoir ${ }^{23}$, M-A.Bizouard ${ }^{19}$, D.Bloch $^{10}$, M.Blume ${ }^{52}$, T.Bolognese ${ }^{39}$, M.Bonesini ${ }^{28}$, W.Bonivento ${ }^{28}$, P.S.L.Booth ${ }^{22}$, C.Bosio ${ }^{40}$, O.Botner $^{48}$, E.Boudinov $^{31}$, B.Bouquet ${ }^{19}$, C.Bourdarios ${ }^{9}$, T.J.V.Bowcock ${ }^{22}$, M.Bozzo ${ }^{13}$, P.Branchini ${ }^{40}$, K.D.Brand ${ }^{36}$, T.Brenke $^{52}$, R.A.Brenner ${ }^{15}$, C.Bricman ${ }^{2}$, R.C.A.Brown ${ }^{9}$, P.Bruckman ${ }^{18}$, J-M.Brunet ${ }^{8}$, L.Bugge ${ }^{33}$, T.Buran ${ }^{33}$, T.Burgsmueller ${ }^{52}$, P.Buschmann ${ }^{52}$, S.Cabrera ${ }^{49}$, M.Caccia $^{28}$, M.Calvi ${ }^{28}$, A.J.Camacho Rozas ${ }^{41}$, T.Camporesi $^{9}$, V.Canale $^{38}$, M.Canepa ${ }^{13}$, K.Cankocak ${ }^{44}$,F.Cao ${ }^{2}$,F.Carena ${ }^{9}$, L.Carroll ${ }^{22}$, C.Caso ${ }^{13}$, M.V.Castillo Gimenez ${ }^{49}$, A.Cattai $^{9}$, F.R.Cavallo ${ }^{5}$, V.Chabaud ${ }^{9}$, M.Chapkin ${ }^{42}$, Ph.Charpentier ${ }^{9}$, L.Chaussard ${ }^{25}$, P.Checchia ${ }^{36}$, G.A.Chelkov ${ }^{16}$, M.Chen ${ }^{2}$, R.Chierici ${ }^{45}$, P.Chliapnikov ${ }^{42}$, P.Chochula ${ }^{7}$, V.Chorowicz ${ }^{9}$, J.Chudoba ${ }^{30}$, V.Cindro ${ }^{43}{ }^{2}$ P.Collins $^{9}$, R.Contri ${ }^{13}$, E.Cortina ${ }^{49}$, G.Cosme ${ }^{19}$, F.Cossutti ${ }^{46}$, J-H.Cowell ${ }^{22}$, H.B.Crawley ${ }^{1}$, D.Crennell ${ }^{37}$, G.Crosetti $^{13}$, J.Cuevas Maestro ${ }^{34}$, S.Czellar ${ }^{15}$, E.Dahl-Jensen ${ }^{29}$, J.Dahm ${ }^{52}$, B.Dalmagne ${ }^{19}$, M.Dam ${ }^{29}$, G.Damgaard $^{29}$, P.D.Dauncey ${ }^{37}$, M.Davenport ${ }^{9}$, W.Da Silva ${ }^{23}$, C.Defoix ${ }^{8}$, A.Deghorain ${ }^{2}$, G.Della Ricca ${ }^{46}$, P.Delpierre $^{27}$, N.Demaria ${ }^{35}$, A.De Angelis ${ }^{9}, \quad$ W.De Boer ${ }^{17}$, S.De Brabandere ${ }^{2}$, C.De Clercq ${ }^{2}$, C.De La Vaissiere ${ }^{23}$, B.De Lotto ${ }^{46}$, A.De Min $^{36}$, L.De Paula ${ }^{47}$, C.De Saint-Jean ${ }^{39}$, H.Dijkstra ${ }^{9}$, L.Di Ciaccio ${ }^{38}$, A.Di Diodato $^{38}$, F.Djama ${ }^{10}$, A.Djannati ${ }^{8}$, J.Dolbeau ${ }^{8}$, K.Doroba ${ }^{51}$, M.Dracos ${ }^{10}$, J.Drees ${ }^{52}$, K.-A.Drees ${ }^{52}$, M.Dris $^{32}$, J-D.Durand ${ }^{25,9}$, D.Edsall ${ }^{1}$, R.Ehret ${ }^{17}$, G.Eigen ${ }^{4}$, T.Ekelof ${ }^{48}$, G.Ekspong ${ }^{44}$, M.Elsing ${ }^{9}$, J-P.Engel ${ }^{10}$, B.Erzen ${ }^{43}$, M.Espirito Santo ${ }^{21}$, E.Falk ${ }^{24}$, D.Fassouliotis ${ }^{32}$, M.Feindt ${ }^{9}$, A.Fenyuk ${ }^{42}$, A.Ferrer ${ }^{49}$, S.Fichet ${ }^{23}$, T.A.Filippas ${ }^{32}$, A.Firestone ${ }^{1}$, P.-A.Fischer ${ }^{10}$, H.Foeth ${ }^{9}$, E.Fokitis ${ }^{32}$, F.Fontanelli ${ }^{13}$, F.Formenti ${ }^{9}$, B.Franek ${ }^{37}$, P.Frenkiel $^{8}$, D.C.Fries ${ }^{17}$, A.G.Frodesen ${ }^{4}$, R.Fruhwirth ${ }^{50}$, F.Fulda-Quenzer ${ }^{19}$, J.Fuster ${ }^{49}$, A.Galloni ${ }^{22}$,

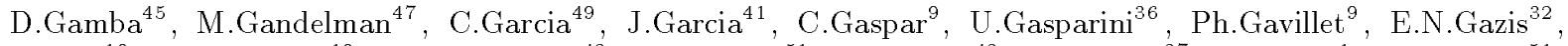
D.Gele ${ }^{10}$, J-P.Gerber ${ }^{10}$, L.Gerdyukov ${ }^{42}$, R.Gokieli ${ }^{51}$, B.Golob ${ }^{43}$, G.Gopal ${ }^{37}$, L.Gorn ${ }^{1}$, M.Gorski ${ }^{51}$, Yu.Gouz ${ }^{45,53}$, V.Gracco ${ }^{13}$, E.Graziani ${ }^{40}$, C.Green $^{22}$, A.Grefrath ${ }^{52}$, P.Gris ${ }^{39}$, G.Grosdidier ${ }^{19}$, K.Grzelak ${ }^{51}$, S.Gumenyuk $^{28,53}$, P.Gunnarsson ${ }^{44}$, M.Gunther ${ }^{48}$, J.Guy ${ }^{37}$, F.Hahn ${ }^{9}$, S.Hahn ${ }^{52}$, Z.Hajduk ${ }^{18}$, A.Hallgren ${ }^{48}$, K.Hamacher $^{52}$, F.J.Harris ${ }^{35}$, V.Hedberg ${ }^{24}$, R.Henriques ${ }^{21}$, J.J.Hernandez ${ }^{49}$, P.Herquet ${ }^{2}$, H.Herr ${ }^{9}$, T.L.Hessing $^{35}$, J.-M.Heuser ${ }^{52}$, E.Higon ${ }^{49}$, H.J.Hilke ${ }^{9}$, T.S.Hill ${ }^{1}$, S-O.Holmgren ${ }^{44}$, P.J.Holt ${ }^{35}$, D.Holthuizen ${ }^{31}$, S.Hoorelbeke ${ }^{2}$, M.Houlden ${ }^{22}$, J.Hrubec ${ }^{50}$, K.Huet ${ }^{2}$, K.Hultqvist ${ }^{44}$, J.N.Jackson ${ }^{22}$, R.Jacobsson ${ }^{44}$, P.Jalocha ${ }^{18}$, R.Janik $^{7}$, Ch.Jarlskog ${ }^{24}$, G.Jarlskog ${ }^{24}$, P.Jarry ${ }^{39}$, B.Jean-Marie ${ }^{19}$, E.K.Johansson ${ }^{44}$, L..Jonsson ${ }^{24}$, P.Jonsson ${ }^{24}$, C.Joram $^{9}$, P.Juillot ${ }^{10}$, M.Kaiser ${ }^{17}$, F.Kapusta ${ }^{23}$, K.Karafasoulis ${ }^{11}$, M.Karlsson ${ }^{44}$, E.Karvelas ${ }^{11}$, S.Katsanevas ${ }^{3}$, E.C.Katsoufis ${ }^{32}$, R.Keranen ${ }^{4}$, Yu.Khokhlov ${ }^{42}$, B.A.Khomenko ${ }^{16}$, N.N.Khovanski ${ }^{16}$, B.King ${ }^{22}$, N.J.Kjaer ${ }^{31}$, O.Klapp ${ }^{52}$, H.Klein ${ }^{9}$, A.Klovning ${ }^{4}$, P.Kluit ${ }^{31}$, B.Koene ${ }^{31}$, P.Kokkinias ${ }^{11}$, M.Koratzinos ${ }^{9}$, K.Korcyl ${ }^{18}$, V.Kostioukhine $^{42}$, C.Kourkoumelis ${ }^{3}$, O.Kouznetsov ${ }^{13,16}$, M.Krammer $^{50}$, C.Kreuter ${ }^{9}$, I.Kronkvist ${ }^{24}$, Z.Krumstein $^{16}$, W.Krupinski ${ }^{18}$, P.Kubinec ${ }^{7}$, W.Kucewicz ${ }^{18}$, K.Kurvinen ${ }^{15}$, C.Lacasta ${ }^{49}$, I.Laktineh ${ }^{25}$, J.W.Lamsa ${ }^{1}$, L.Lanceri ${ }^{46}$, D.W.Lane ${ }^{1}$, P.Langefeld ${ }^{52}$, V.Lapin ${ }^{42}$, J-P.Laugier ${ }^{39}$, R.Lauhakangas ${ }^{15}$, G.Leder $^{50}$, F.Ledroit $^{14}$, V.Lefebure ${ }^{2}$, C.K.Legan ${ }^{1}$, R.Leitner ${ }^{30}$, J.Lemonne ${ }^{2}$, G.Lenzen ${ }^{52}$, V.Lepeltier ${ }^{19}$, T.Lesiak ${ }^{18}$, J.Libby $^{35}$, D.Liko ${ }^{9}$, R.Lindner ${ }^{52}$, A.Lipniacka ${ }^{44}$, I.Lippi ${ }^{36}$, B.Loerstad ${ }^{24}$, J.G.Loken ${ }^{35}$, J.M.Lopez ${ }^{41}$, D.Loukas $^{11}$, P.Lutz ${ }^{39}$, L.Lyons ${ }^{35}$, J.MacNaughton ${ }^{50}$, G.Maehlum ${ }^{17}$, J.R.Mahon ${ }^{6}$, A.Maio ${ }^{21}$, T.G.M.Malmgren ${ }^{44}$, V.Malychev ${ }^{16}$, F.Mandl ${ }^{50}$, J.Marco ${ }^{41}$, R.Marco ${ }^{41}$, B.Marechal ${ }^{47}$, M.Margoni $^{36}$, J-C.Marin $^{9}$, C.Mariotti ${ }^{9}$, A.Markou ${ }^{11}$, C.Martinez-Rivero ${ }^{34}$, F.Martinez-Vidal ${ }^{49}$, S.Marti i Garcia ${ }^{22}$, J.Masik ${ }^{30}$, F.Matorras $^{41}$, C.Matteuzzi ${ }^{28}$, G.Matthiae ${ }^{38}$, M.Mazzucato ${ }^{36}$, M.Mc Cubbin ${ }^{22}$, R.Mc Kay ${ }^{1}$, R.Mc Nulty ${ }^{22}$, J.Medbo $^{48}$, M.Merk ${ }^{31}$, C.Meroni ${ }^{28}$, S.Meyer ${ }^{17}$, W.T.Meyer ${ }^{1}$, M.Michelotto ${ }^{36}$, E.Migliore ${ }^{45}$, L.Mirabito ${ }^{25}$, W.A.Mitaroff ${ }^{50}$, U.Mjoernmark ${ }^{24}$, T.Moa ${ }^{44}$, R.Moeller ${ }^{29}$, K.Moenig ${ }^{9}$, M.R.Monge ${ }^{13}$, P.Morettini ${ }^{13}$, H.Mueller $^{17}$, K.Muenich ${ }^{52}$, M.Mulders ${ }^{31}$, L.M.Mundim ${ }^{6}$, W.J.Murray ${ }^{37}$, B.Muryn ${ }^{14,18}$, G.Myatt ${ }^{35}$, F.Naraghi ${ }^{14}$, F.L.Navarria ${ }^{5}$, S.Navas ${ }^{49}$, K.Nawrocki ${ }^{51}$, P.Negri ${ }^{28}$, S.Nemecek ${ }^{12}$, W.Neumann ${ }^{52}$, R.Nicolaidou ${ }^{3}$, B.S.Nielsen ${ }^{29}$, M.Nieuwenhuizen $^{31}$, V.Nikolaenko ${ }^{10}$, P.Niss ${ }^{44}$, A.Nomerotski ${ }^{36}$, A.Normand ${ }^{35}$, W.Oberschulte-Beckmann ${ }^{17}$, V.Obraztsov ${ }^{42}$, A.G.Olshevski ${ }^{16}$, A.Onofre ${ }^{21}$, R.Orava ${ }^{15}$, K.Osterberg ${ }^{15}$, A.Ouraou ${ }^{39}$, P.Paganini ${ }^{19}$, M.Paganoni ${ }^{9,28}$, P.Pages ${ }^{10}$, R.Pain ${ }^{23}$, H.Palka ${ }^{18}$, Th.D.Papadopoulou ${ }^{32}$, K.Papageorgiou ${ }^{11}$, L.Pape ${ }^{9}$, C.Parkes $^{35}$, F.Parodi ${ }^{13}$, A.Passeri ${ }^{40}$, M.Pegoraro ${ }^{36}$, L.Peralta ${ }^{21}$, H.Pernegger ${ }^{50}$, M.Pernicka ${ }^{50}$, A.Perrotta ${ }^{5}$, C.Petridou $^{46}$, A.Petrolini ${ }^{13}$, M.Petrovyck ${ }^{42}$, H.T.Phillips ${ }^{37}$, G.Piana ${ }^{13}$, F.Pierre ${ }^{39}$, M.Pimenta ${ }^{21}$, T.Podobnik ${ }^{43}$, O.Podobrin ${ }^{9}$, M.E.Pol ${ }^{6}$, G.Polok ${ }^{18}$, P.Poropat ${ }^{46}$, V.Pozdniakov ${ }^{16}$, P.Privitera ${ }^{38}$, N.Pukhaeva ${ }^{16}$, A.Pullia ${ }^{28}$, D.Radojicic ${ }^{35}$, S.Ragazzi ${ }^{28}$, H.Rahmani ${ }^{32}$, P.N.Ratoff ${ }^{20}$, A.L.Read ${ }^{33}$, M.Reale $^{52}$, P.Rebechi $^{19}$, N.G.Redaelli ${ }^{28}$, M.Regler ${ }^{50}$, D.Reid ${ }^{9}$, R.Reinhardt ${ }^{52}$, P.B.Renton ${ }^{35}$, L.K.Resvanis ${ }^{3}$, F.Richard ${ }^{19}$, J.Richardson ${ }^{22}$, J.Ridky ${ }^{12}$, G.Rinaudo $^{45}$, I.Ripp ${ }^{39}$, A.Romero ${ }^{45}$, I.Roncagliolo ${ }^{13}$, P.Ronchese ${ }^{36}$, L.Roos ${ }^{23}$, E.I.Rosenberg ${ }^{1}$, P.Roudeau $^{19}$, T.Rovelli ${ }^{5}$, W.Ruckstuhl ${ }^{31}$, V.Ruhlmann-Kleider ${ }^{39}$, A.Ruiz ${ }^{41}$, K.Rybicki ${ }^{18}$, H.Saarikko ${ }^{15}$, Y.Sacquin ${ }^{39}$, A.Sadovsky ${ }^{16}$, O.Sahr ${ }^{14}$, G.Sajot ${ }^{14}$, J.Salt ${ }^{49}$, J.Sanchez ${ }^{26}$, M.Sannino ${ }^{13}$, M.Schimmelpfennig ${ }^{17}$, H.Schmeider ${ }^{17}$, U.Schwickerath $^{17}$, M.A.E.Schyns ${ }^{52}$, G.Sciolla ${ }^{45}$, F.Scuri ${ }^{46}$, P.Seager ${ }^{20}$, Y.Sedykh ${ }^{16}$, A.M.Segar ${ }^{35}$, A.Seitz ${ }^{17}$, R.Sekulin ${ }^{37}$, L.Serbelloni ${ }^{38}$, R.C.Shellard ${ }^{6}$, P.Siegrist ${ }^{39}$, R.Silvestre ${ }^{39}$, S.Simonetti ${ }^{39}$, F.Simonetto $^{36}$, A.N.Sisakian ${ }^{16}$, B.Sitar ${ }^{7}$, T.B.Skaali ${ }^{33}$, G.Smadja $^{25}$, N.Smirnov ${ }^{42}$, O.Smirnova ${ }^{24}$, G.R.Smith ${ }^{37}$, R.Sosnowski ${ }^{51}$, D.Souza-Santos ${ }^{6}$, T.Spassov ${ }^{21}$, E.Spiriti ${ }^{40}$, P.Sponholz ${ }^{52}$, S.Squarcia ${ }^{13}$, D.Stampfer ${ }^{9}$, C.Stanescu ${ }^{40}$, S.Stanic ${ }^{43}$, S.Stapnes $^{33}$, I.Stavitski ${ }^{36}$, K.Stevenson ${ }^{35}$, A.Stocchi ${ }^{19}$, J.Strauss ${ }^{50}$, R.Strub ${ }^{10}$, B.Stugu ${ }^{4}$, M.Szczekowski ${ }^{51}$, 
M.Szeptycka $^{51}$, T.Tabarelli ${ }^{28}$, J.P.Tavernet ${ }^{23}$, O.Tchikilev ${ }^{42}$, J.Thomas ${ }^{35}$, A.Tilquin ${ }^{27}$, J.Timmermans ${ }^{31}$, L.G.Tkatchev ${ }^{16}$, T.Todorov ${ }^{10}$, S.Todorova ${ }^{10}$, D.Z.Toet ${ }^{31}$, A.Tomaradze ${ }^{2}$, A.Tonazzo ${ }^{28}$, L.Tortora ${ }^{40}$, G.Transtromer ${ }^{24}$, D.Treille ${ }^{9}$, G.Tristram ${ }^{8}$, A.Trombini ${ }^{19}$, C.Troncon ${ }^{28}$, A.Tsirou ${ }^{9}$, M-L.Turluer ${ }^{39}$, I.A.Tyapkin ${ }^{16}$, M.Tyndel $^{37}$, S.Tzamarias ${ }^{22}$, B. Ueberschaer ${ }^{52}$, O.Ullaland ${ }^{9}$, V.Uvarov ${ }^{42}$, G.Valenti ${ }^{5}$, E.Vallazza ${ }^{9}$, $\begin{array}{ll}\text { C.Vander Velde } & \text { G.W.Van Apeldoorn } \\ & \end{array}$, P.Van Dam ${ }^{31}$, W.K.Van Doninck ${ }^{2}$ J.Van Eldik ${ }^{31}$, A.Van Lysebetten ${ }^{2}$, N.Vassilopoulos ${ }^{35}$, G.Vegni ${ }^{28}$, L.Ventura ${ }^{36}$, W.Venus ${ }^{37}$, F.Verbeure ${ }^{2}$, M.Verlato $^{36}$, L.S.Vertogradov $^{16}$, D.Vilanova ${ }^{39}$, P.Vincent ${ }^{25}$, L.Vitale $^{46}$, E.Vlasov ${ }^{42}$, A.S.Vodopyanov ${ }^{16}$, V.Vrba $^{12}$, H.Wahlen ${ }^{52}$, C.Walck ${ }^{44}$, M.Weierstall ${ }^{52}$, P.Weilhammer ${ }^{9}$, C.Weiser ${ }^{17}$, A.M.Wetherell ${ }^{9}$, D.Wicke ${ }^{52}$, J.H.Wickens ${ }^{2}$, M.Wielers ${ }^{17}$, G.R.Wilkinson ${ }^{9}$, W.S.C.Williams ${ }^{35}$, M.Winter ${ }^{10}$, M.Witek ${ }^{18}$, T.Wlodek ${ }^{19}$, K.Woschnagg $^{48}$, K.Yip ${ }^{35}$, O.Yushchenko ${ }^{42}$, F.Zach ${ }^{25}$, A.Zaitsev ${ }^{42}$, A.Zalewska ${ }^{9}$, P.Zalewski ${ }^{51}$, D.Zavrtanik ${ }^{43}$, E.Zevgolatakos ${ }^{11}$, N.I.Zimin ${ }^{16}$, M.Zito $^{39}$, D.Zontar ${ }^{43}$, G.C.Zucchelli ${ }^{44}$, G.Zumerle $^{36}$

\footnotetext{
${ }^{1}$ Department of Physics and Astronomy, Towa State University, Ames IA 50011-3160, USA

${ }^{2}$ Physics Department, Univ. Instelling Antwerpen, Universiteitsplein 1, B-2610 Wilrijk, Belgium and IIHE, ULB-VUB, Pleinlaan 2, B-1050 Brussels, Belgium

and Faculté des Sciences, Univ. de l'Etat Mons, Av. Maistriau 19, B-7000 Mons, Belgium

${ }^{3}$ Physics Laboratory, University of Athens, Solonos Str. 104, GR-10680 Athens, Greece

${ }^{4}$ Department of Physics, University of Bergen, Allégaten 55, N-5007 Bergen, Norway

${ }^{5}$ Dipartimento di Fisica, Università di Bologna and INFN, Via Irnerio 46, I-40126 Bologna, Italy

${ }^{6}$ Centro Brasileiro de Pesquisas Físicas, rua Xavier Sigaud 150, RJ-22290 Rio de Janeiro, Brazil and Depto. de Física, Pont. Univ. Católica, C.P. 38071 RJ-22453 Rio de Janeiro, Brazil and Inst. de Física, Univ. Estadual do Rio de Janeiro, rua São Francisco Xavier 524, Rio de Janeiro, Brazil ${ }^{7}$ Comenius University, Faculty of Mathematics and Physics, Mlynska Dolina, SK-84215 Bratislava, Slovakia

${ }^{8}$ Collège de France, Lab. de Physique Corpusculaire, IN2P3-CNRS, F-75231 Paris Cedex 05, France

${ }^{9} \mathrm{CERN}, \mathrm{CH}-1211$ Geneva 23, Switzerland

${ }^{10}$ Centre de Recherche Nucléaire, IN2P3 - CNRS/ULP - BP20, F-67037 Strasbourg Cedex, France

${ }^{11}$ Institute of Nuclear Physics, N.C.S.R. Demokritos, P.O. Box 60228, GR-15310 Athens, Greece

${ }^{12}$ FZU, Inst. of Physics of the C.A.S. High Energy Physics Division, Na Slovance 2, 180 40, Praha 8, Czech Republic

${ }^{13}$ Dipartimento di Fisica, Università di Genova and INFN, Via Dodecaneso 33, I-16146 Genova, Italy

${ }^{14}$ Institut des Sciences Nucléaires, IN2P3-CNRS, Université de Grenoble 1, F-38026 Grenoble Cedex, France

${ }^{15}$ Research Institute for High Energy Physics, SEFT, P.O. Box 9, FIN-00014 Helsinki, Finland

${ }^{16}$ Joint Institute for Nuclear Research, Dubna, Head Post Office, P.O. Box 79, 101000 Moscow, Russian Federation

${ }^{17}$ Institut für Experimentelle Kernphysik, Universität Karlsruhe, Postfach 6980, D-76128 Karlsruhe, Germany

${ }^{18}$ Institute of Nuclear Physics and University of Mining and Metalurgy, Ul. Kawiory 26a, PL-30055 Krakow, Poland

${ }^{19}$ Université de Paris-Sud, Lab. de l'Accélérateur Linéaire, IN2P3-CNRS, Bât. 200, F-91405 Orsay Cedex, France

${ }^{20}$ School of Physics and Chemistry, University of Lancaster, Lancaster LA1 4YB, UK

${ }^{21}$ LIP, IST, FCUL - Av. Elias Garcia, 14-1 ${ }^{\circ}$, P-1000 Lisboa Codex, Portugal

${ }^{22}$ Department of Physics, University of Liverpool, P.O. Box 147, Liverpool L69 3BX, UK

${ }^{23}$ LPNHE, IN2P3-CNRS, Universités Paris VI et VII, Tour 33 (RdC), 4 place Jussieu, F-75252 Paris Cedex 05, France

${ }^{24}$ Department of Physics, University of Lund, Sölvegatan 14, S-22363 Lund, Sweden

${ }^{25}$ Université Claude Bernard de Lyon, IPNL, IN2P3-CNRS, F-69622 Villeurbanne Cedex, France

${ }^{26}$ Universidad Complutense, Avda. Complutense s/n, E-28040 Madrid, Spain

${ }^{27}$ Univ. d'Aix - Marseille II - CPP, IN2P3-CNRS, F-13288 Marseille Cedex 09, France

${ }^{28}$ Dipartimento di Fisica, Università di Milano and INFN, Via Celoria 16, I-20133 Milan, Italy

${ }^{29}$ Niels Bohr Institute, Blegdamsvej 17, DK-2100 Copenhagen 0, Denmark

${ }^{30}$ NC, Nuclear Centre of MFF, Charles University, Areal MFF, V Holesovickach 2, 180 00, Praha 8, Czech Republic

${ }^{31}$ NIKHEF, Postbus 41882, NL-1009 DB Amsterdam, The Netherlands

${ }^{32}$ National Technical University, Physics Department, Zografou Campus, GR-15773 Athens, Greece

${ }^{33}$ Physics Department, University of Oslo, Blindern, N-1000 Oslo 3, Norway

${ }^{34}$ Dpto. Fisica, Univ. Oviedo, Avda. Calvo Sotelo, S/N-33007 Oviedo, Spain, (CICYT-AEN96-1681)

${ }^{35}$ Department of Physics, University of Oxford, Keble Road, Oxford OX1 3RH, UK

${ }^{36}$ Dipartimento di Fisica, Università di Padova and INFN, Via Marzolo 8, I-35131 Padua, Italy

${ }^{37}$ Rutherford Appleton Laboratory, Chilton, Didcot OX11 OQX, UK

${ }^{38}$ Dipartimento di Fisica, Università di Roma II and INFN, Tor Vergata, I-00173 Rome, Italy

${ }^{39} \mathrm{CEA}$, DAPNIA/Service de Physique des Particules, CE-Saclay, F-91191 Gif-sur-Yvette Cedex, France

${ }^{40}$ Istituto Superiore di Sanità, Ist. Naz. di Fisica Nucl. (INFN), Viale Regina Elena 299, I-00161 Rome, Italy

${ }^{41}$ Instituto de Fisica de Cantabria (CSIC-UC), Avda. los Castros, S/N-39006 Santander, Spain, (CICYT-AEN96-1681)

${ }^{42}$ Inst. for High Energy Physics, Serpukov P.O. Box 35, Protvino, (Moscow Region), Russian Federation

${ }^{43}$ Depart ment of Astroparticle Physics, School of Environmental Sciences, Nova Gorica, and J. Stefan Institute, Ljubljana, Slovenia

${ }^{44}$ Fysikum, Stockholm University, Box 6730, S-113 85 Stockholm, Sweden

${ }^{45}$ Dipartimento di Fisica Sperimentale, Università di Torino and INFN, Via P. Giuria 1, I-10125 Turin, Italy

${ }^{46}$ Dipartimento di Fisica, Università di Trieste and INFN, Via A. Valerio 2, I-34127 Trieste, Italy and Istituto di Fisica, Università di Udine, I-33100 Udine, Italy

${ }^{47}$ Univ. Federal do Rio de Janeiro, C.P. 68528 Cidade Univ., Tha do Fundão BR-21945-970 Rio de Janeiro, Brazil

${ }^{48}$ Department of Radiation Sciences, University of Uppsala, P.O. Box 535, S-751 21 Uppsala, Sweden

${ }^{49}$ IFIC, Valencia-CSIC, and D.F.A.M.N., U. de Valencia, Avda. Dr. Moliner 50, E-46100 Burjassot (Valencia), Spain

${ }^{50}$ Institut für Hochenergiephysik, Österr. Akad. d. Wissensch., Nikolsdorfergasse 18, A-1050 Vienna, Austria

${ }^{51}$ Inst. Nuclear Studies and University of Warsaw, Ul. Hoza 69, PL-00681 Warsaw, Poland

${ }^{52}$ Fachbereich Physik, University of Wuppertal, Postfach 100 127, D-42097 Wuppertal, Germany

${ }^{53}$ On leave of absence from IHEP Serpukhov
} 


\section{Introduction}

The existence of Neutral Heavy Leptons $\left(\nu_{m}\right)$ which couple to the $Z^{0}$ boson is a prediction of several models proposed as alternatives to the Standard Model [1]. Some of these schemes, such as the left-right symmetric and see-saw models [2], constitute electroweak extensions to the Standard Model and incorporate these new particles as a possible explanation of the neutrino mass puzzle. In these scenarios, the difference between the mass scales for leptons and neutrinos is understood in terms of accompanying massive right-handed neutrino partners which are weak isospin singlets $[3,4]$.

In $\mathrm{e}^{+} \mathrm{e}^{-}$interactions, single production of such particles can occur through production of the $Z^{0}$ resonance and its subsequent decay into a standard anti-neutrino and its Neutral Heavy Lepton partner, i.e. $\mathrm{e}^{+} \mathrm{e}^{-} \rightarrow \mathrm{Z}^{0} \rightarrow \nu_{m} \bar{\nu}$. The branching ratio for this process can be expressed as [4]:

$$
B R\left(\mathrm{Z}^{0} \rightarrow \nu_{m} \bar{\nu}\right)=B R\left(\mathrm{Z}^{0} \rightarrow \nu \bar{\nu}\right)|U|^{2}\left(1-\frac{m_{\nu_{m}}^{2}}{m_{\mathrm{Z}^{0}}{ }^{2}}\right)^{2}\left(1+\frac{1}{2} \frac{m_{\nu_{m}}^{2}}{m_{\mathrm{Z}^{0}}^{2}}\right)
$$

where $U$ is a mixing matrix element and $B R\left(Z^{0} \rightarrow \nu \bar{\nu}\right) \simeq 0.063$ is the branching ratio for a single neutrino species. There is another similar decay, $Z^{0} \rightarrow \bar{\nu}_{m} \nu$, with the same branching ratio.

Constraints arising from weak universality [3] provide an upper bound of order $10^{-2}$ on $|U|^{2}$. The above processes should be the dominant production modes as the corresponding $Z^{0} \rightarrow \nu_{m} \overline{\nu_{m}}$ pair production cross section is suppressed by an additional $|U|^{2}$ factor. The mean decay length of these particles $(L)$ is a function of $|U|^{2}$ and the mass $m_{\nu_{m}}$. For the $\mathrm{Z}^{0} \rightarrow \nu_{m} \bar{\nu}$ process at LEP I, this dependence can be approximated by [3]:

$$
L \approx \frac{3}{|U|^{2}\left(m_{\nu_{m}}\left(\mathrm{GeV} / c^{2}\right)\right)^{6}}(\mathrm{~cm})
$$

For $m_{\nu_{m}}$ equal to $3 \mathrm{GeV} / c^{2}$ or less, the decay of such an object may thus be detected far from the interaction point ( $\sim 100 \mathrm{~cm}$ or more) if one is sensitive to branching ratios of order $10^{-6}$ or less. In view of the high statistics available from LEP I, exploring long mean decay lengths is therefore necessary in order to extend the limits to the lowest possible values of $m_{\nu_{m}}$.

In all these models, Neutral Heavy Leptons decay weakly via the neutral $\left(Z^{0}\right)$ or charged $\left(\mathrm{W}^{ \pm}\right)$currents according to [4]:

$$
\begin{aligned}
& \nu_{m} \rightarrow \nu \quad \mathrm{Z}^{*} \\
& \longrightarrow \nu \bar{\nu}, \ell \bar{l}, \mathrm{q} \bar{q} \\
& \nu_{m} \rightarrow \ell^{\prime} \mathrm{W}^{*} \\
& \longrightarrow \nu \bar{\ell}, \mathrm{q} \bar{q}^{\prime}
\end{aligned}
$$

with $\ell=e, \mu, \tau, q=u, d, s, c, b$, and $q \bar{q}^{\prime}=u \bar{d}, c \bar{s}$; and including charge conjugate states.

This gives four different decay topologies $\left(\nu \nu \bar{\nu}, \nu \ell \bar{\ell}, \nu \mathrm{q} \bar{q}\right.$ and $\left.\ell q \bar{q}^{\prime}\right)$ with branching ratios that depend only weakly on the Neutral Heavy Lepton mass. For masses below $50 \mathrm{GeV} / c^{2}$ the charged current contribution is around $75 \%$, increasing to $80 \%$ for masses around $75 \mathrm{GeV} / \mathrm{c}^{2}$, and the branching ratios are rather constant and take the values [4]: $B R\left(\nu_{m} \rightarrow \nu \nu \bar{\nu}\right)=0.06, B R\left(\nu_{m} \rightarrow \nu \ell \bar{\ell}\right)=0.24, B R\left(\nu_{m} \rightarrow \nu \mathrm{q} \bar{q}\right)=0.15$, and $B R\left(\nu_{m} \rightarrow\right.$ $\left.\ell \bar{q}^{\prime}\right)=0.55$. 
The large amount of data collected at LEP I allows searches for Neutral Heavy Leptons in the various possible event topologies as a function of the mass and lifetime. For small masses, less than about $30 \mathrm{GeV} / c^{2}$, the experimental signature is a monojet (a single cluster of particles), due to the large boost of the $\nu_{m}$. For higher masses, the predominant decay into a $q \bar{q}$ pair and a lepton gives two jets, acollinear and acoplanar with respect to the beam axis, with or without an associated charged lepton depending on the decay channel. In both cases, the events are characterised by the large missing energy and momentum carried by the neutrinos. The searches based on these signatures assume the $\nu_{m}$ has a short lifetime. To cover longer lifetimes, searches are made based on displaced vertices and calorimeter clusters.

The short-lifetime signatures are also shared by other processes, like the production of a light Higgs boson $\left(\mathrm{e}^{+} \mathrm{e}^{-} \rightarrow H \nu \bar{\nu}\right)$, light neutralinos $\left(\mathrm{e}^{+} \mathrm{e}^{-} \rightarrow \tilde{\chi}_{1}^{0} \tilde{\chi}_{2}^{0}\right.$ with $\left.\tilde{\chi}_{2}^{0} \rightarrow \tilde{\chi}_{1}^{0} Z^{*}\right)$, or composite excited neutrinos $\nu^{*}$. For example, the results of this analysis can be extended to the case of single production of excited neutrinos when standard $\mathrm{SU}(2) \times \mathrm{U}(1)$ currents are assumed. Then the only $\nu^{*}$ decay channels allowed are $\nu^{*} \rightarrow \ell W^{*}$ and $\nu^{*} \rightarrow \nu Z^{*}$, since the coupling $\gamma \nu \nu^{*}$ vanishes. Single production of excited neutrinos [5] at LEP I energies is given by:

$$
\Gamma\left(Z^{0} \rightarrow \nu^{*} \nu\right)=\frac{1}{3} \alpha m_{\mathrm{Z}^{0}}{ }^{3}\left(\frac{c_{Z \nu^{*} \nu}}{\Lambda}\right)^{2}\left(1-\frac{m_{\nu^{*}}^{2}}{m_{\mathrm{Z}^{0}}{ }^{2}}\right)^{2}\left(1+2 \frac{m_{\nu^{*}}^{2}}{m_{\mathrm{Z}^{0}}{ }^{2}}\right)
$$

where $c_{Z \nu^{*} \nu}$ is the coupling of the $Z^{0}$ to the neutrino and its excited partner, and $\Lambda$ is the compositeness scale. The $\nu^{*}$ lifetime is expected to be very short.

Searches for heavy neutrals produced in $e^{+} e^{-}$collisions have been unsuccessful in the past [6], although the LEP experiments have found some interesting events. In particular, ALEPH have reported three monojet events [7] with a probability, in the Standard Model, of less than $0.1 \%$.

Neutral Heavy Leptons have also been searched for in leptonic decays of hadrons and in neutrino beam experiments [8], leading to stringent upper limits on $|U|^{2}$ reaching $10^{-7}$ in the low mass region $\left(m_{\nu_{m}}\right.$ below $\left.2-3 \mathrm{GeV} / c^{2}\right)$. Two LEP experiments, OPAL and L3, have also placed limits on the branching ratio $B R\left(Z^{0} \rightarrow \nu_{m} \bar{\nu}\right)$ of the order of $10^{-4}-10^{-5}$ for masses above $3 \mathrm{GeV} / c^{2}[9]$.

The data and experimental techniques presented in this paper improve on the previous sensitivities in this higher mass range by about one order of magnitude.

The paper is organised as follows. Section 2 describes the experimental procedure and gives a brief description of the different data samples used. Sections 3 to 6 describe the different analysis methods used. Finally, the combined results are presented in section 7 .

\section{Experimental procedure and event sample}

The analysis is based on data collected by DELPHI at LEP I from 1991 to 1994 inclusive, corresponding to $3.3 \times 10^{6}$ hadronic $Z^{0}$ decays and a total sample of $\sim 12.3 \times 10^{6}$ events recorded on tape. Detailed descriptions of the apparatus, the trigger conditions, the event processing chain, and the detector performance are given in references [10-12].

Fig. 1 shows a schematic view of the detector with the angular coverage of the different subsystems. The Neutral Heavy Lepton search has been performed in the barrel region of the detector, for polar angles $(\theta)$ between $45^{\circ}$ and $135^{\circ}$. Due to the specific nature of the signal, namely events with missing energy and momentum, all of the information 
available from the detector has to be used in order to discriminate against the various background processes that could mimic a signal.

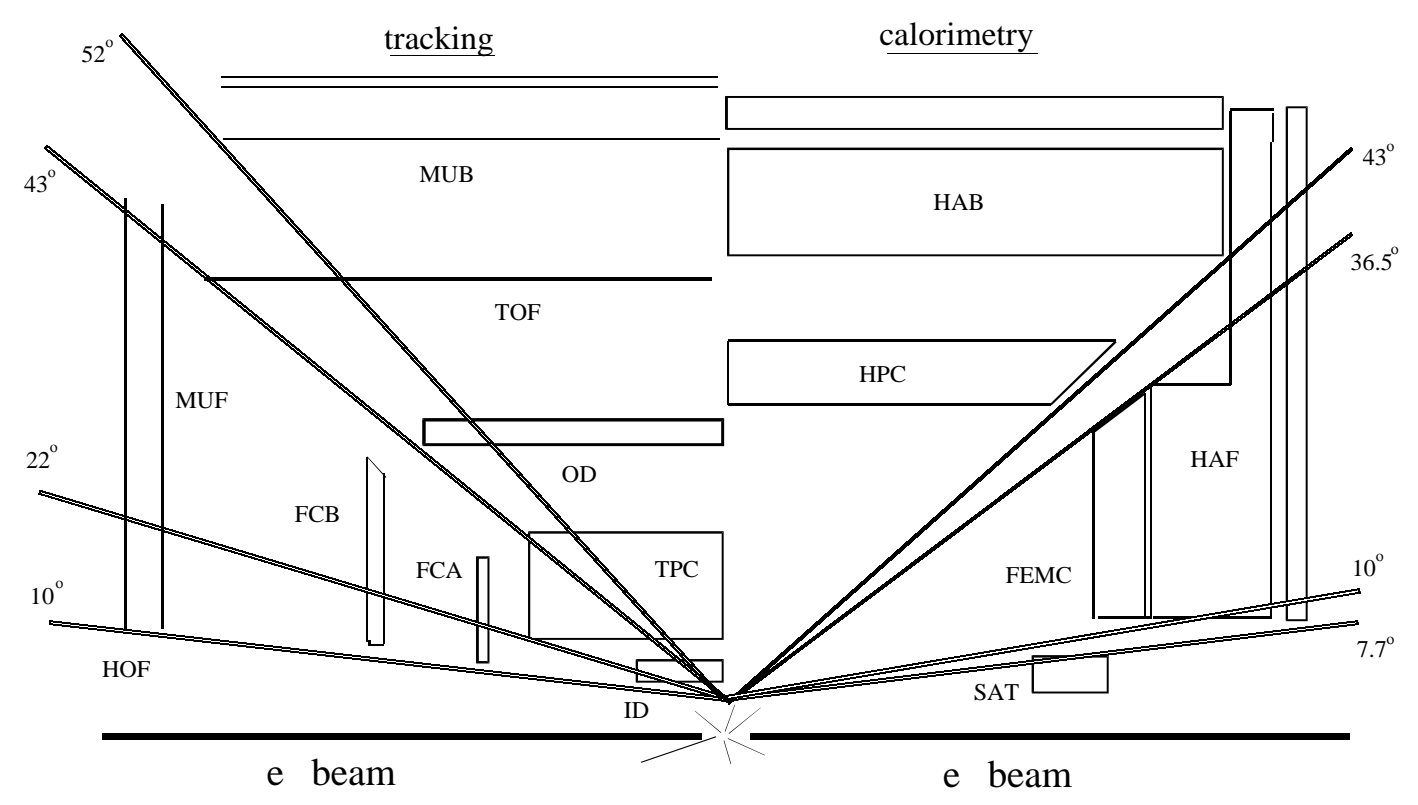

Figure 1: Schematic view of the 1991-1993 DELPHI detector. The left of the picture depicts the tracking devices, together with their angular coverage. The calorimeters are shown on the right. Standard acronyms denote the detectors: VD (Vertex Detector), ID (Inner detector), TPC (Time Projection Chamber), OD (Outer Detector), TOF (Time Of Flight), MUB (MUon chambers in the Barrel region), FCA and FCB (Forward Chambers A and B), MUF (MUon chambers in the Forward region), HOF (scintillator HOdoscope in the Forward region), HPC (High density Projection Chamber), HAB and HAF (HAdronic calorimeter in the Barrel and Forward regions), FEMC (Forward ElectroMagnetic Calorimeter) and SAT (Small Angle Tagger). In 1994, the SAT was replaced by the STIC (Scintillating TIle Calorimeter) which has a larger angular acceptance overlapping that of the FEMC.

The present analysis covers the range of $\nu_{m}$ masses from $2 m_{\mu}$ up to the kinematic limit $m_{\mathrm{Z}^{0}}$. To achieve this coverage, the possibility of very long $\nu_{m}$ decay lengths has to be considered, as remarked above. This analysis includes, for the first time, searches for $\nu_{m}$ decays at any distance within the active detector volume [13]. Four different searches have been performed:

- Short lifetimes and small masses : Events originating from the reaction $Z^{0} \rightarrow$ $\bar{\nu} \nu_{m}$ and the subsequent $\nu_{m}$ decay within $12 \mathrm{~cm}$ of the interaction point (IP) are characterised by large missing momentum and a monojet-like topology (section 3 ).

- Short lifetimes and large masses : In the mass range $40-80 \mathrm{GeV} / c^{2}$, the $\nu_{m}$ necessarily has a very short lifetime, and decays predominantly into a hadronic system composed of acoplanar and acollinear jets (section 4).

- Intermediate lifetimes : For $\nu_{m}$ decays occurring from $12 \mathrm{~cm}$ to $110 \mathrm{~cm}$, the most characteristic feature is an isolated set of charged particle tracks which originate from the same vertex (section 5).

- Long lifetimes : When the $\nu_{m}$ decays at radii from $110 \mathrm{~cm}$ to $300 \mathrm{~cm}$, in the detector region where charged particle tracks cannot be reconstructed, the search 
has to rely on localized clusters of energy depositions and hits in the outermost layers of the detector. The inefficiency of this method for detecting unresolved pairs of electrons sets the lower limit mentioned above of $2 m_{\mu}$ to the mass range explored (see section 6).

In order to compute the detection efficiencies, a total of $10^{5}$ signal events in the different $\nu_{m}$ decay channels with $\nu_{m}$ masses ranging from $1.5 \mathrm{GeV} / c^{2}$ to $85 \mathrm{GeV} / c^{2}$ and mean decay lengths from $0 \mathrm{~cm}$ up to $2000 \mathrm{~cm}$ were generated using JETSET [14] and passed through the full DELPHI detector simulation [15]. All three types of $\nu_{m}\left(\nu_{m e}, \nu_{m \mu}, \nu_{m \tau}\right)$ and their decays have been included when kinematically allowed.

Standard generators $[14,16]$ were used to estimate the backgrounds (see Table 1 ) from $\mathrm{q} \bar{q}(\gamma), \mathrm{e}^{+} \mathrm{e}^{-}(\gamma), \mu^{+} \mu^{-}(\gamma), \tau^{+} \tau^{-}(\gamma)$ and from $\gamma \gamma$ processes. The background from fourfermion final state processes $\left(\mathrm{e}^{+} \mathrm{e}^{-} \rightarrow f \bar{f} \nu \bar{\nu}, f \equiv q, e, \mu, \tau\right)$ was evaluated using the program EXCALIBUR [17].

\begin{tabular}{|l|c|c|c|c|c|c|c|c|c|}
\hline \multirow{2}{*}{ Sample } & \multicolumn{4}{|c|}{$\gamma \gamma$} & 4-fermion & \multicolumn{4}{|c|}{$Z^{0}$ decays } \\
\cline { 2 - 7 } & $\mathrm{e}^{+} \mathrm{e}^{-}$ & $\mu^{+} \mu^{-}$ & $\tau^{+} \tau^{-}$ & $\mathrm{q} \overline{\mathrm{q}}$ & & $\mathrm{e}^{+} \mathrm{e}^{-}$ & $\mu^{+} \mu^{-}$ & $\tau^{+} \tau^{-}$ & $\mathrm{q} \overline{\mathrm{q}}$ \\
\hline \# Generated & 27256 & 99316 & 9984 & 62079 & 14700 & 118835 & 1417494 & 411902 & 4333000 \\
\cline { 2 - 7 } \# Expected & 39614 & 94110 & 30389 & 68083 & 1067 & 143230 & 143230 & 143230 & 2979699 \\
\hline
\end{tabular}

Table 1: Sizes of the simulated event samples used to estimate the backgrounds.

\section{Low mass and short lifetime $\nu_{m}$ : Search for mono- jets}

The monojet candidates were selected by requiring a single jet of particles and no energy depositions in the direction opposite to the jet. Only neutral showers with energy larger than $500 \mathrm{MeV}$ and charged particles with momentum above $100 \mathrm{MeV} / \mathrm{c}$ were considered. Furthermore, a charged particle track was required to have a length of at least $30 \mathrm{~cm}$, and a distance of closest approach to the interaction point of less than 5 $\mathrm{cm}$ in the plane perpendicular to the beam ( $R \phi$ plane, where $R$ is the radial coordinate and $\phi$ the azimuthal angle) and less than $10 \mathrm{~cm}$ along the beam direction $(z)$. When computing invariant masses, the tracks were given the mass provided by the standard DELPHI particle identification tools [10] when possible, otherwise they were assumed to be pions. This criterion has been applied in all the analyses described in the paper. Two sets of requirements were applied to the original sample of events accepted by the trigger.

1. General requirements to suppress the low energy background arising from beam-gas, beam-wall and $\gamma \gamma$ interactions:

- the total energy in the event had to exceed $5 \mathrm{GeV}$ and the total energy carried by the charged particles had to exceed $3 \mathrm{GeV}$,

- there had to be at least one charged particle with $p>2.5 \mathrm{GeV} / c$,

- no charged particle track or electromagnetic shower with $|\cos \theta| \geq 0.96$ was allowed, where $\theta$ is the polar angle,

- no single energy deposition in the FEMC above $10 \mathrm{GeV}$ was allowed.

After this selection, $3.9 \times 10^{6}$ events remained. 
2. Specific requirements for $\nu_{m}$ topologies, whose main features are a considerable amount of missing momentum and energy:

- $p_{t \text { miss }} / E_{\text {vis }}$ had to exceed $\max \left(-0.4\left[\frac{m_{e v t}^{2}}{70^{2}}-1\right], 0.2\right)$, where $p_{t \text { miss }}$ is the projection of the missing momentum of the event in the $R \phi$ plane, $E_{\mathrm{vis}}$ is the total visible energy, and $m_{e v t}$ is the total invariant mass; this cut implicitly rejected events with missing momentum pointing to very forward regions and small invariant masses, such as $\gamma \gamma$ interactions, and it also rejected normal $\mathrm{Z}^{0}$ decays, which usually have very small $p_{t \text { miss }}$ and large values of $E_{\text {vis }}$ and $m_{\text {evt }}$ (Fig. 2), - $p_{t_{\text {miss }}}$ had to exceed $4.5 \mathrm{GeV} / c$,

- the largest angle between any two particles with $E \geq 500 \mathrm{MeV}$ had to be less than $160^{\circ}$,

- the energy inside a cone of $80^{\circ}$ half opening angle around the missing momentum direction had to be less than $300 \mathrm{MeV}$,

- the first measured point of at least $70 \%$ of all charged particle tracks had to lie within $12 \mathrm{~cm}$ of the interaction point: this cut rejected events which contained photon conversions or interactions inside the detector.

After this further selection, 211 events remained.

For the surviving events, the cluster finding algorithm LUCLUS [14] was applied to the charged particles. The cut-off parameter used by this algorithm, $d_{\text {join }}$, was set to 8 $\mathrm{GeV} / \mathrm{c}$ in order to allow for high jet masses. Events were accepted if only one jet with more than one charged particle track was reconstructed with total momentum larger than $3 \mathrm{GeV} / c$ and $\left|\cos \theta_{\mathrm{j} e t}\right|$ was below 0.8 , where $\theta_{\text {jet }}$ was the polar angle of the jet. Events with two reconstructed jets were also accepted if at least one of the jets fulfilled these conditions on momentum and direction and the angle between the jets was less than $140^{\circ}$. One-particle jets were allowed in 2-jet events, in order to cope with the $\nu \ell \bar{\ell}$ and $\ell q \bar{q}^{\prime}$ decay topologies.

At this stage, 114 events were left in the analysis, while 133 were expected from the simulations of standard background processes, namely $\gamma \gamma$ events, $Z^{0}$ decays, and four fermion final states.

The background events were expected to be mainly $\gamma \gamma$ interactions or $Z^{0}$ leptonic decays with radiative photons or charged particles escaping through inefficient regions of the detector. To reduce this background, events were rejected if the missing momentum vector was pointing at polar angle regions where the detector coverage was weak $\left(33^{\circ} \leq\right.$ $\theta \leq 43^{\circ}, 89^{\circ} \leq \theta \leq 91^{\circ}$, and $\left.137^{\circ} \leq \theta \leq 147^{\circ}\right)^{\dagger}$. This cut left 81 events in the real data sample, to be compared with 85 background events expected from the simulation.

The $\gamma \gamma$ background events that contributed at this stage corresponded to a well defined topology which contained visible charged particle tracks in the barrel region, one electron missing in the gap at $\theta \simeq 9^{\circ}$ between the SAT and FEMC detectors (see Fig. 1), and the other electron escaping undetected inside the beam pipe. In order to eliminate such events, the direction of the electron missing at wider angle was calculated using the reconstructed energy and momentum and assuming the other electron (or positron) was inside the beam pipe and carrying $\sim 45 \mathrm{GeV} / \mathrm{c}$ momentum: events were rejected if the calculated electron direction was pointing to the region between the SAT and the FEMC. This cut rejected 35 events, in agreement with 41 events expected from the simulation. No such background was present in the data sample collected in 1994 since the STIC, which had replaced the SAT, overlaps with the FEMC. Thus all $\gamma \gamma$ events were eliminated from the simulated sample at this stage.

\footnotetext{
${ }^{\dagger}$ At LEP-II these regions are covered by dedicated photon taggers, but these were not yet installed for the LEP-I data analysed here.
} 
In addition to this background, $\tau^{+} \tau^{-}$final states also contribute to the selected sample. This happens when one $\tau$ decays into several charged particles and the other $\tau$ produces one charged particle which traverses a dead zone of the detector, or has a momentum below the minimum required in the selection of charged particle tracks $(100 \mathrm{MeV} / \mathrm{c})$. The latter events were identified using the information from the ID and VD, since the three layers of the VD allowed the momentum cut-off to be reduced below $50 \mathrm{MeV} / \mathrm{c}$. To reduce the $\tau$ background further, the events with a three-charged-particle monojet were required to have an opening angle greater than $10^{\circ}$ or a mass greater than $5 \mathrm{GeV} / \mathrm{c}^{2}$. This cut removed all the remaining $\tau^{+} \tau^{-}$background events from the simulated data sample and left just one event in the real data.

The surviving event, shown in Fig. 3, had two well isolated electrons with an invariant mass of $300 \mathrm{MeV} / c^{2}$ and missing $p_{t}$ of $6 \mathrm{GeV} / c$. Both electron tracks were reconstructed as originating from the interaction point and they are not consistent with coming from the same point in the beam pipe or further out; so they are unlikely to be due to a real photon conversion. The invariant mass and the transverse momentum of this event are compatible with the four-fermion process $e^{+} e^{-} \rightarrow f \bar{f} \nu \bar{\nu}(f \equiv q, e, \mu, \tau)$. The number of background events expected from that process evaluated using EXCALIBUR amounted to 0.5 events in the $l \bar{l} \nu \bar{\nu}$ channel and 0.3 in the $q \bar{q} \nu \bar{\nu}$ channel. The contaminations from $\gamma \gamma$ processes and $Z^{0}$ decays were also estimated from the simulation samples but were found to be negligible.

Thus the total number of background events expected was 0.8 , while one event was observed. This event can be interpreted as due to the standard electroweak four-fermion reaction $e^{+} e^{-} \rightarrow e^{+} e^{-} \nu \bar{\nu}$, but for the limit calculations it was regarded as a candidate.

Applying the above selection to the simulated signal events gave the efficiencies for different masses as shown in Fig. 4 (dashed curve). Trigger and acceptance efficiencies were taken into account. The trigger efficiency approaches $100 \%$ on account of the high multiplicity in the case of high masses and the high transverse momentum of the isolated leptons for masses below $40 \mathrm{GeV} / c^{2}$ [13]. The maximum overall efficiency of this search was $\sim 50 \%$, obtained for masses of $2-15 \mathrm{GeV} / c^{2}$.

\section{High mass and short lifetime $\nu_{m}$ : Search for acollinear jets}

This analysis considered a singly produced $\nu_{m}$, in the high mass range from $40 \mathrm{GeV} / \mathrm{c}^{2}$ to $80 \mathrm{GeV} / c^{2}$. In this mass range the heavy neutrino is assumed to have a very short lifetime (see Eq. 2) and to decay predominantly into a $q \bar{q}^{\prime}$ pair and a lepton (see Eqs. 3 and 4). The main signature of such events is a hadronic system consisting of acollinear and acoplanar jets, with or without an associated charged lepton depending on the decay channel.

The main background is hadronic $Z^{0}$ decays with missing energy and momentum due to particles escaping detection (ordinary neutrinos, etc.) or particles lost in regions of the detector with poor coverage. In order to discriminate against this background a large number of variables were needed. Neural networks were therefore used as a part of the selection procedure.

Charged particles with momenta greater than $100 \mathrm{MeV} / \mathrm{c}$ and neutrals with energies above $100 \mathrm{MeV}$ were selected to compute the event variables used. The event topology was classified according to three different methods: the first method divided the event into 
two hemispheres with respect to the plane perpendicular to the thrust axis, the second method performed a jet search using the cluster algorithm LUCLUS [14] with $d_{\text {join }}=2.5$ $\mathrm{GeV} / c$ (default value), and in the third method LUCLUS was forced to reconstruct three jets.

First, the following preselection of events was applied in order to remove the bulk of the background events in both the neutral current NC (Eq. 3) and charged current CC (Eq. 4) channels:

- the total number of charged particles was required to be greater than 7 ,

- the transverse projection of the total missing momentum was required to be greater than $5 \mathrm{GeV} / c$,

- the total observed energy was required to be greater than $20 \mathrm{GeV}$ for the NC analysis and $25 \mathrm{GeV}$ for the $\mathrm{CC}$ analysis,

- the total reconstructed invariant mass had to fall in the interval $10 \mathrm{GeV} / c^{2}<$ $m_{e v t}<75 \mathrm{GeV} / c^{2}(\mathrm{NC})$ or $35 \mathrm{GeV} / c^{2}<m_{e v t}<75 \mathrm{GeV} / c^{2}(\mathrm{CC})$,

- the acollinearity angle was required to be larger than $7^{\circ}$ for the $\mathrm{NC}$ analysis and $10^{\circ}$ for the CC analysis,

- the acoplanarity angle was required to be greater than $15^{\circ}$ (only for the $\mathrm{NC}$ analysis),

- the maximum angle between any two jets was required to be less than $160^{\circ}$ for the $\mathrm{NC}$ analysis and $175^{\circ}$ for the $\mathrm{CC}$ analysis,

- the minimum angle between any jet and the missing momentum direction was required to be larger than $20^{\circ}$ (CC analysis only),

- the sum of the angles between the jets, when forcing three jets, was required to be smaller than $357^{\circ}$.

These preselection criteria were chosen to maximize the signal efficiency while rejecting the simulated background samples, consisting of hadronic $Z^{0}$ decays, and four-fermion and $\gamma \gamma$ processes with hadronic final states (see table 1 ). The event preselection reduced the $\mathrm{q} \overline{\mathrm{q}}$ background sample by factors of 460 and 230 with efficiencies close to $65 \%$ and $70 \%$ for the neutral current (NC) and charged current (CC) analysis, respectively.

The next step was the neural network selection. The networks were of the feed-forward type, with back-propagation of errors [18]. They had 15 input nodes, two intermediate layers of 8 and 5 nodes respectively, one output node, and a total of 165 degrees of freedom. The networks were trained to give output close to zero for a background event surviving the preselection, and close to one for a signal event. Independent signal and $q \bar{q}$ background samples were used for the training, which was terminated after 3500 cycles through the samples. The network parameters (weights) were then chosen from the training cycle which maximized the signal to background ratio for network outputs above 0.9. A detailed description of the network can be found in [19].

For each of the decay channels (charged and neutral current), two analyses were optimised for $m_{\nu_{m}}=50 \mathrm{GeV} / c^{2}$ and $65 \mathrm{GeV} / c^{2}$, respectively, in order to maximize the overall efficiency in the range $40-80 \mathrm{GeV} / \mathrm{c}^{2}$. The final selection in each channel was the logical OR of the two analyses. The overall efficiency as a function of $m_{\nu_{m}}$ was obtained by applying the selection to the $\nu_{m}$ samples simulated with different masses $(40,50,60,65$, 70 , and $\left.80 \mathrm{GeV} / \mathrm{c}^{2}\right)$.

The preselected simulated background events were then passed through the neural networks, and the cut on the network output variable was made. This rejected all of the simulated $\gamma \gamma$ background events, but 30 simulated $q \bar{q}$ events and 5 simulated four-fermion events remained in the OR of the two analyses in all decay channels combined. 
Further cuts were necessary in order to discriminate against this remaining background. These selections were different for the different decay channels and for the different masses used in the optimization, and were classified as follows.

- Hermeticity cuts (left 22 q $\bar{q}$ and 3 four-fermion events) were applied in order to reject events with particles probably going towards less instrumented regions of the detector. Events were rejected if the polar angle of the missing momentum direction was close to $90^{\circ}$ or $40^{\circ}$ or $140^{\circ}$, see the discussion in section 3 .

- Event quality cuts (left $6 \mathrm{q} \bar{q}$ and 1 four-fermion events) rejected events with more than $20 \%$ of the total reconstructed energy coming from badly reconstructed tracks, or with total measured energy within a $40^{\circ}$ cone around the missing momentum direction greater than $500 \mathrm{MeV}$. A badly reconstructed track is a charged particle track which does not fulfil the standard DELPHI hadronic track selection described in $[10]$.

- Topological cuts (removed all the remaining simulated background events) were applied using global shape variables not used by the network:

- the transverse projection of the minimum angle between any jet and the missing momentum direction was required to be larger than $10^{\circ}$,

- the transverse projection of the maximum of the angles between any two jets was required to be smaller than $170^{\circ}$,

- the cosine of the angle between the sphericity axis and the boost direction calculated in the c.m.s. of the observed particles was required to be smaller than 0.8 ,

- the maximum angle between any two jets when forcing three jets was required to be smaller than $170^{\circ}$,

- and the acoplanarity angle was required to be larger than $5^{\circ}$.

The above selections left no simulated $q \bar{q}, \gamma \gamma$, or four-fermion event in any of the subanalyses.

When the same selection was applied to the real data accumulated from 1991 to 1994 , no event was selected. The total signal efficiency obtained, as a function of the $\nu_{m}$ mass, is shown in Fig. 4 (dotted curve). Trigger and acceptance efficiencies are taken into account (see the discussion at the end of previous section).

\section{$5 \quad$ Intermediate lifetime $\nu_{m}$ : Vertex search using tracking}

A neutral particle that decays within the radial distance region $12 \mathrm{~cm}<\mathrm{R}<110 \mathrm{~cm}$ can be detected as a reconstructed secondary vertex by the central tracking devices, ID and TPC. To suppress low energy background, the general criteria used in the monojet search, section 3 , were imposed prior to vertex reconstruction but with looser cuts in the track selection. Cosmic ray events were rejected by requiring at least 1 hit in the TOF within $12 \mathrm{~ns}(3 \sigma)$ of the beam collision time. For $\mathrm{Z}^{0} \rightarrow \mu^{+} \mu^{-}$events, the efficiency of this timing selection was calculated to be $\sim 88 \%$ averaged over all different data acquisition periods. This sets a minimum efficiency for the timing selection for a single minimum ionising particle of $65 \%$. After these cuts, $4.4 \times 10^{6}$ events remained.

A cluster finding algorithm was then applied to the remaining events in order to group the charged particle tracks according to their first measured point ('starting point'). This procedure was iterative and worked as follows. The pair of tracks with the smallest 
separation at their respective starting points was considered first. If this separation was smaller than $10 \mathrm{~cm}$, the tracks were grouped to form a cluster whose starting point was defined as the average of their first measured points. The two tracks were then replaced by this cluster, which was subsequently treated as a pseudo-track. The process was then repeated until all charged particle tracks or pseudo-tracks were grouped into clusters.

Events were rejected if no cluster was found or the starting points of two or more clusters were closer than $12 \mathrm{~cm}$ from the nominal interaction point. After these selections. $2.6 \times 10^{6}$ events remained.

The vertex reconstruction method of [20] was then applied to each cluster of charged particle tracks. The efficiency of this method, within the ID and TPC volume, was determined by the simulation to be around $70 \%$ to $80 \%$ depending on the $\nu_{m}$ mass, between 5 and $85 \mathrm{GeV} / \mathrm{c}^{2}$, and on the mean decay length, between 20 and $500 \mathrm{~cm}$. Each vertex was defined by its radius vector $(\vec{V})$ calculated with respect to the interaction point. In addition, the total momentum $\left(\overrightarrow{\mathrm{P}}_{\vec{V}}\right)$ and the invariant mass $\left(\mathrm{m}_{\vec{V}}\right)$, were calculated from the four-momenta of the charged particles belonging to the vertex. Two vertices were combined into a single one if the angle between their radius vectors was smaller than the largest opening angle in either cluster.

Events were then rejected if no vertex was reconstructed, if two or more vertices were closer than $12 \mathrm{~cm}$ from the interaction point, or if one vertex was found closer than 1 $\mathrm{cm}$ from the interaction point. These requirements left 192872 events, mostly cosmic ray events, beam-gas or beam-wall interactions, badly reconstructed $\mathrm{Z}^{0}$ decays and gamma conversions.

In order to select events with isolated vertices of the type expected from a $\nu_{m}$ decay, a selection based on $\vec{V}, \vec{P}_{\vec{V}}$, and $m_{\vec{V}}$ was applied. Events were accepted if at least one vertex passed the following selections:

1. cuts defining the geometrical region and acceptance (left 7715 events):

- $|\vec{V}|$ had to be above $12 \mathrm{~cm}$,

- $\left|\cos \left(\theta_{\vec{V}}\right)\right|$ had to be below 0.766 ,

2. vertex quality cuts (left 3858 events):

- $\left|\overrightarrow{\mathrm{P}}_{\overrightarrow{\mathrm{V}}}\right|$ had to be above $3 \mathrm{GeV} / c$,

- at least one charged particle track belonging to the vertex had to have a momentum larger than $1.5 \mathrm{GeV} / c$,

- it was required that no hits were found in the VD inside a $5^{\circ}$ cone around the direction of $\vec{V}$,

3. rejection of cosmic rays (left 1929 events):

- the angle between $\vec{P}_{\vec{V}}$ and $\vec{V}$ had to be smaller than $60^{\circ}$,

- the impact parameter with respect to the interaction point of the line defined by the vertex and $\vec{P}_{\vec{V}}$ had to be less than $75 \mathrm{~cm}$,

- the vertex had to open towards the outside of the DELPHI detector,

4. rejection of vertices coming from photon conversions (left 965 events):

- for events with two charged particles, $\mathrm{m}_{\vec{v}}$ had to exceed $0.75 \mathrm{GeV} / c^{2}$,

5. isolation criteria and rejection of back-to-back events (no event left):

- it was required that no other vertex was found,

- charged particle tracks which could not be associated to the vertex had to be outside a $45^{\circ}$ half angle cone around $\overrightarrow{\mathrm{V}}$, 
- charged particle tracks in a $30^{\circ}$ half angle cone around the direction opposite to $\vec{V}$ had to have less than $75 \%$ of the total energy and momentum in that hemisphere, and any isolated neutral showers in the opposite hemisphere energy had to have total energy less than $10 \mathrm{GeV}$.

The efficiencies for different mean decay lengths $(L)$ and masses $\left(m_{\nu_{m}}\right)$ were found for each $\nu_{m}$ decay mode by applying the above selections to the simulated signal samples (see Figs. 5 and 6, dotted curves). Trigger and acceptance efficiencies have been taken into account. For the trigger efficiency, values above $80 \%$ are expected since the full calorimetric trigger is still active. The reduction in the efficiency with respect to that for the short lifetime search is due to the fact that there is less redundancy in the tracking trigger. The maximum efficiency $(\sim 25 \%)$ was obtained for masses below $20 \mathrm{GeV} / c^{2}$ and mean decay lengths around $50 \mathrm{~cm}$.

No events in the real data sample were found to satisfy all the conditions described above.

\section{Long lifetime $\nu_{m}$ : Search for calorimeter clusters}

The cluster method was developed in order to search for long-lived $\nu_{m}$ whose decay products interacted with the outermost layers of DELPHI, i.e. TOF, HAB, and MUB. If there was information present from the inner tracking detectors, it was also used as it contributed efficiently to the rejection of in-time cosmic ray muons traversing the detector close to the interaction point, which is the main reason to confine the analysis to the barrel $\left(40^{\circ}<\theta<140^{\circ}\right)$. The use of the cluster information also increased the detection efficiency for $\nu_{m}$ decays inside the tracking devices when the low track multiplicity did not enable the vertex reconstruction.

The main signature of a $\nu_{m}$ decaying in the outer parts of DELPHI is a cluster of hits, in time with the beam collision, confined within a relatively small angular region and pointing back to the interaction point. Therefore, the general strategy to search for a $\nu_{m}$ in this region was based on the TOF timing information, used as in the vertex analysis (previous section) to reduce the cosmic background, and on the spatial distribution of the hit coordinates provided by the detectors.

The hit information from the TOF, HAB and MUB was used to select events in the $R \phi$ plane. For each event, the maximum and minimum azimuthal angles $\left(\phi_{\max }, \phi_{\min }\right)$ among the TOF, HAB and MUB hits were defined. A $\nu_{m}$ decay is characterized by a small difference between $\phi_{\max }$ and $\phi_{\min }$. For cosmic ray muons, these two angles are much less correlated. Similarly, correlations between maximum and minimum $z$ coordinates $\left(z_{\max }\right.$, $\left.z_{\text {min }}\right)$ were analyzed using HAB and MUB hits. Again, similar values for $z_{\max }$ and $z_{\min }$ are the main feature of a $\nu_{m}$ decay.

A first selection, based on the TOF timing and on the event topology as described above, reduced the data sample to 35721 events. In order to reject standard $Z^{0}$ decays, the following conditions were applied :

- the total number of reconstructed charged particle tracks had to be less than 3 ,

- all charged particle tracks in the event had to start at a radial distance $R$ greater than $12 \mathrm{~cm}$ from the interaction point,

- events with two charged particle tracks had to have an acollinearity larger than $5^{\circ}$,

- the charged particle track with the highest momentum had not to point within $\pm 1^{\circ}$ of any of the 6 TPC sector boundaries. 
These cuts removed an additional $80 \%$ of the real data. The remaining events were mainly cosmic ray muons, $Z^{0}$ leptonic events with particles escaping undetected through inefficient regions of the detector, and events with two or more real photons.

To reduce these kinds of backgrounds further, tighter cuts on the event topology were imposed. Two different categories of events were distinguished, namely those with hits in the MUB ( $\mu$-like) and those with energy deposited in the HAB $(\pi$-like). For $\mu$-like events, restrictive conditions were imposed to ensure that the hits were consistent with being caused by the decay of a neutral particle originating from the interaction point. This was done by fitting all hits to a trajectory, then calculating the impact parameter of the trajectory. The event was rejected if the impact parameter was above $40 \mathrm{~cm}$ in both the $R \phi$ and the $z$ projection. These cuts are not as stringent as in the previous analysis due to the larger errors in this case. For $\pi$-like events, the energy depositions in the HAB were required to be larger than the expectation for a single muon and consistent with a hadronic shower. This left 323 events in the data, mainly cosmic ray muons not rejected by the TOF timing selection and entering the detector either tangentially (giving hits only in the HAB and TOF detectors) or with small impact parameters, and two-photon final states and dilepton events with particles escaping detection. These were rejected in the final three steps of the analysis:

- Veto against two photon final states (left 234 events):

Events with two real photons usually have electromagnetic energy deposited in the HPC, a back-to-back configuration, and no hits in the MUB or the HAB. However, sometimes their orientation coincides with cracks in the HPC and they reach the HAB, depositing large amounts of energy therein. Such events were rejected if the energy reconstructed in the HPC was close to a crack in this detector or was greater than $35 \mathrm{GeV}$ for each photon.

- Veto against cosmic ray events (left 15 events):

To eliminate the remaining cosmic ray muons, the longitudinal profile of the shower in the HAB was used. Events were regarded as cosmic ray muons if:

- the energy profile in $\mathrm{HAB}$ along the reconstructed trajectory was compatible with that expected from a muon ( there was energy deposited in all the calorimeter layers),

- there were MUB hits associated to the trajectory within $10^{\circ}$ in the $R \phi$ projection,

- no energy deposition or hits were found outside a $5^{\circ}$ cone around the trajectory in the $R \phi$ projection.

The possibility of two parallel cosmic muons crossing the detector was also taken into account. Two parallel trajectories which both satisfied the above conditions were rejected.

- Veto against dilepton events (left no events):

Dilepton events with one lepton entering a TPC crack were identified by matching TPC sector boundaries with back-to-back signal configurations in the MUB or the $\mathrm{HPC}$ or with hits in the three layers of the VD.

The efficiencies were derived for the different decay channels as a function of the mass and the decay length (as shown in Figs. 5 and 6 , dashed curves) by applying the same selection to the simulated signal events. Trigger and acceptance efficiencies have also been calculated [13] and taken into account. The trigger efficiency is strongly dependent on the mass and the decay length. For a $20 \mathrm{GeV} / c^{2} \nu_{m}$, it is above $60 \%$ for a mean decay length of $500 \mathrm{~cm}$ and above $35 \%$ for one of $1000 \mathrm{~cm}$. The maximum efficiency, $15 \%$ for 
a mean decay length of $100 \mathrm{~cm}$, was obtained for masses around $3 \mathrm{GeV} / \mathrm{c}^{2}$. In the case of $\nu_{m} \rightarrow \nu \ell \vec{\ell}$, the method was sensitive to decays inside the tracking devices, since low multiplicity events were accepted.

No event in the real data sample was selected with the above criteria.

\section{Summary and Conclusions}

Searches have been made for short-lived and for long-lived Neutral Heavy Leptons using several different methods. No evidence for the existence of Neutral Heavy Leptons has been found, and limits at the $95 \%$ confidence level (CL) on their production are therefore derived below. For high masses, the limits are calculated using the $\nu_{m}$ decay branching ratios quoted in the introduction. For masses below $2 \mathrm{GeV} / c^{2}$, the charged current contribution starts decreasing and it equals the neutral current one at around $1 \mathrm{GeV} / c^{2}$. However, the final results are not significantly affected by variations in the branching ratios, since the sensitivity is very similar for all channels. In the low mass region, only the kinematically allowed channels, with light leptons and quarks, have been taken into account.

\subsection{Short-lived Neutral Heavy Leptons}

Two searches have been performed for short-lived Neutral Heavy Leptons. The first method, the search for monojet events, was sensitive to low $\nu_{m}$ masses. The second, searching for events with acollinear and acoplanar jets, was optimised for high $\nu_{m}$ masses. By combining these two searches, the full mass range from the kinematic threshold up to $m_{Z^{\circ}}$ was explored. Taking acceptance and trigger efficiency into account, an overall efficiency of nearly 50\% was obtained, as shown in Fig. 4. One candidate event was found (in the monojet analysis). It was interpreted as a standard four fermion reaction, $e^{+} e^{-} \rightarrow e^{+} e^{-} \nu \bar{\nu}$, but was retained when computing the limits.

Fig. 7 shows the limit on the branching ratio $B R\left(Z^{0} \rightarrow \nu_{m} \bar{\nu}\right)$ obtained, while the corresponding limits on the mixing parameter $|U|^{2}$ are shown as a function of $m_{\nu_{m}}$ by the right-most exclusion area in Fig 10. For masses between 6 and $50 \mathrm{GeV} / c^{2}$, the branching ratio was found to be limited to $B R\left(Z^{0} \rightarrow \nu_{m} \bar{\nu}\right)<1.3 \times 10^{-6}$ at $95 \% \mathrm{CL}$, which corresponds to $|U|^{2}<2.1 \times 10^{-5}$ in the middle of the mass range. In computing the limits, the efficiencies have been corrected for the fraction of events in which the $\nu_{m}$ decays outside the region of interest $(R<12 \mathrm{~cm})$. This correction is important only for $m_{\nu_{m}}$ values below $5 \mathrm{GeV} / c^{2}$, which yield large decay lengths according to Eq. 2.

\subsection{Long-lived Neutral Heavy Leptons}

Two searches have also been performed for long-lived Neutral Heavy Leptons, the first for $\nu_{m}$ decaying in the tracking volume (vertex search) and the second for $\nu_{m}$ decaying in the outer layers of the detector (cluster search). No candidate events were found in either search.

The vertex search investigated the mass range where the particle would have a detectable lifetime and decay in the region where the tracking chambers of DELPHI are effective. The cluster method, optimised for low masses, was sensitive to even longer lifetimes and larger mean decay lengths. Figs. 5 and 6 show the efficiencies of these methods as a function of the mean decay length and the $m_{\nu_{m}}$ for each possible decay mode. A 
small overlap exists between the two searches which was considered when computing the overall efficiency. The maximum overall efficiency after combining the two searches was $\sim 30 \%$.

For a long-lived Neutral Heavy Lepton, limits on $B R\left(Z^{0} \rightarrow \nu_{m} \bar{\nu}\right)$ as a function of $m_{\nu_{m}}$ and the mean decay length were obtained from the vertex and cluster methods (Fig.8), yielding upper limits smaller than $3 \times 10^{-6}$ for mean decay lengths below $1000 \mathrm{~cm}$.

However, due to the relationship between $m_{\nu_{m}}$ and the mean $\nu_{m}$ decay length $(L)$, given by Eq. 2, not all pairs of $\left(m_{\nu_{m}}, L\right)$ values are permitted theoretically. Taking this constraint into account, for a given $m_{\nu_{m}}$, only the mean decay lengths for which the experimental upper limit on the branching ratio is bigger than the theoretical prediction of Eq. 2 are allowed by the data, as indicated in Fig. 9a. The corresponding region of excluded $\left(m_{\nu_{m}}, L\right)$ pairs is shown in Fig. $9 \mathrm{~b}$. The resulting limits on $|U|^{2}$ are shown by the left-most exclusion region in Fig. 10.

\subsection{Combination of Short-lived and Long-lived Neutral Heavy Lepton Searches}

Fig. 10 shows the upper limits on $|U|^{2}$ derived from the searches for both short-lived and long-lived Neutral Heavy Leptons. The search for long-lived Neutral Heavy Leptons extends the limit of $1.3 \times 10^{-5}$, obtained from the search for short-lived Neutral Heavy Leptons, from $m_{\nu_{m}} \simeq 6 \mathrm{GeV} / c^{2}$ down to $m_{\nu_{m}} \simeq 3.5 \mathrm{GeV} / c^{2}$. As can be seen from Fig. 11 , where the combination of both analyses is shown, the results obtained from this analysis considerably improve on all previously published limits obtained for $m_{\nu_{m}}$ above $3 \mathrm{GeV} / \mathrm{c}^{2}$.

The limits on $|U|^{2}$ obtained from this analysis apply equally to all flavours of Neutral Heavy Lepton, ie equally to $\left|U_{e}\right|^{2},\left|U_{\mu}\right|^{2}$, and $\left|U_{\tau}\right|^{2}$, except for the consequences of the additional kinematical suppression of the charged current decays in the case of a Neutral Heavy Tau Lepton, due to the large mass of the $\tau$. The main effect is to increase the decay length of such a lepton, leading to limits on $\left|U_{\tau}\right|^{2}$ that are looser by a factor $\simeq 1.5$ for a mass of $3 \mathrm{GeV} / c^{2}$, rising rapidly to a factor 4 for masses of $2 \mathrm{GeV} / c^{2}$ or less, where the mean decay lengths corresponding to the $\left|U^{2}\right|$ limits obtained are very long (see Fig. 9b) and the charged current decay is fully suppressed. It should be noted that the non-LEP limits shown in Fig. 11 generally apply only to $\left|U_{e}\right|^{2}$ and/or $\left|U_{\mu}\right|^{2}$, not to $\left|U_{\tau}\right|^{2}$.

The limits extracted can also be applied to any other hypothetical neutral particle whose characteristics and experimental signature are similar to those studied here. This is the case for single production of excited neutrinos $\left(\nu^{*}\right)$. Equation (5), combined with the above results assuming that only the weak decays $\nu^{*} \rightarrow \ell W^{*}$ and $\nu^{*} \rightarrow \nu Z^{*}$ are allowed, yields a $95 \%$ confidence level upper limit on the parameter $c_{Z \nu^{*} \nu} / \Lambda[13]$ — the ratio between the coupling of $\nu^{*}$ with $\nu$ and $Z^{0}$, and the compositeness scale - as a function of $m_{\nu^{*}}$, as shown in Fig. 12. For masses between 2 and $60 \mathrm{GeV} / c^{2}$, the parameter $c_{Z \nu^{*} \nu} / \Lambda$ is constrained to be less than about $5 \times 10^{-5} \mathrm{GeV}^{-1}$. As shown in Fig. 12, this result improves on previously published LEP I limits based on searches for weak decays. ALEPH [21] and a previous DELPHI analysis [22] investigated the production of $\nu^{*}$ by searching for single photon events, assuming that the coupling $\gamma \nu \nu^{*}$ exists making $\nu^{*} \rightarrow \gamma \nu$ the dominant decay channel. L3 [23] investigated both the electromagnetic decay and the decay through the weak current $\left(\nu^{*} \rightarrow e W\right)$. 


\section{Acknowledgements}

We are greatly indebted to our technical collaborators and to the funding agencies for their support in building and operating the DELPHI detector, and to the members of the CERN-SL division for the excellent performance of the LEP collider. We thank A. Santamaría from the theoretical department of Valencia University for his helpful comments and suggestions concerning the theoretical aspects of the paper and R. Kleiss from the theoretical department at NIKHEF for his help in understanding some aspects of the program EXCALIBUR. 


\section{References}

[1] J.W.F. Valle, 'Weak and electromagnetic interactions in nuclei', Ed. by Klapdor (Springer, Berlin, 1986) 927, and references therein;

J.W.F. Valle, Nucl. Phys. (Proc. Suppl.) 11 (1989) 118.

[2] M. Gell-Mann, P. Ramond, R. Slansky, Supergravity, Ed. by D. Freedman et al. (North Holland, 1979);

T. Yanagida, KEK lectures, Ed. by O. Sawada et al. (1979);

R. Mohapatra, G. Senjanovic, Phys. Rev. Lett. 44 (1980) 912;

R. Mohapatra, G. Senjanovic, Phys. Rev. D23 (1981) 165.

[3] M. Gronau, C.Leung and J. Rosner, Phys. Rev. D29 (1984) 2539.

[4] M. Dittmar, M.C. Gonzalez-García, A. Santamaría, J.W.F Valle, Nucl. Phys. B332 (1990) 1.

[5] E. Boss, A. Pukhov, A. Beliaev, Phys. Lett. B296 (1992) 452;

F. Boudjema and A Djouadi, Phys. Lett. B240 (1990) 485.

[6] JADE Collaboration, W. B. Artel et al., Phys. Lett. B155 (1985) 288;

HRS Collaboration, C. Akerlof et al., Phys. Lett. B156 (1985) 271;

CELLO Collaboration, H. J. Behrend et al., Phys. Lett. B161 (1985) 182;

MARK II Collaboration, G.J. Feldman et al., Phys. Rev. Lett. 54 (1985) 2289;

MAC Collaboration, W. Ash et al., Phys. Rev. Lett. 54 (1985) 2477;

OPAL Collaboration, M.Z. Akrawy et al., Phys. Lett. B236 (1990) 224;

ALEPH Collaboration, D. Decamp et al., Phys. Lett. B236 (1990) 233;

DELPHI Collaboration, P. Abreu et al., Nucl. Phys. B342 (1990) 1;

L3 Collaboration, B. Adeva et al., Phys. Lett. B248 (1990) 203;

OPAL Collaboration, M. Z. Akrawy et al., Phys. Lett. B247 (1990) 448;

L3 Collaboration, O. Adriani et al., Phys. Lett. B295 (1992) 371;

CHARM II Collaboration, P. Vilain et al., Phys. Lett. B295 (1992) 371.

[7] ALEPH Collaboration, D. Buskulic et al., Phys. Lett. B313 (1993) 312;

ALEPH Collaboration, D. Buskulic et al., Phys. Lett. B334 (1994) 224;

ALEPH Collaboration, D. Buskulic et al., Zeit. Phys. C66 (1995) 3.

[8] A. M. Cooper-Sarkar et al., Phys. Lett B160 (1985) 207;

J. Dorenbosch et al., Phys. Lett. B166 (1986) 473;

S. R. Mishra et al., Phys. Rev Lett. 59 (1987) 1397;

M. E. Duffy et al., Phys. Rev. D38 (1988) 2032;

CCFR collaboration, P. de Barbaro et al., A search for Neutral Heavy Leptons in muon-neutrino - N interactions, Proceedings of the Moriond Workshop on New and Exotic Phenomena, January 1990, Les Arcs, France;

CHARM II collaboration, P. Vilain et al., Phys. Lett. B343 (1995) 453, and Phys. Lett. B351 (1995) 387.

[9] OPAL Collaboration, M.Z. Akrawy et al., Phys. Lett. B247 (1990) 448;

L3 Collaboration, O. Adriani et al., Phys. Lett. B295 (1992) 371.

[10] DELPHI Collaboration, P. Abreu et al., Nucl. Instr. and Meth. A303 (1991) 233;

DELPHI Collaboration, P. Abreu et al., Nucl. Instr. and Meth. A378 (1996) 57.

[11] DELPHI Trigger Group, V. Bocci et al., Nucl. Instr. Meth. A362 (1995) 361.

[12] W. Adam et al., IEEE Trans. Nucl. Sci. 39 (1992) 166.

[13] C. Lacasta, Búsqueda de Leptones Neutros Pesados en el detector DELPHI de LEP, Ph.D. Thesis, University of Valencia, IFIC 96-35.

[14] T. Sjöstrand, Comp. Phys. Comm. 82 (1994) 74;

T. Sjöstrand and M. Bengtsson, Comp. Phys. Comm. 46 (1987) 43. 
[15] DELSIM Reference Manual. DELPHI note 89-68 PROG 143, Sep. 1989 (unpublished).

[16] BABAMC: F. A. Berends, W. Hollok and R. Kleiss. Nucl. Phys. B304 (1988) 7112; DYMU3: J. E. Campagne and R. Zitoun, Z. Phys. C43 (1989), and Proc. of the Brighton Workshop on Radiative Corrections, Sussex, July 1989;

KORALZ: S. Jadach et al, Comp. Phys. Comm. 79 (194) 503;

TWOGAM: S. Nova and T. Todorov. DELPHI 90-35 PROG 152 (1990).

[17] F.A. Berends, R. Pittau, R. Kleiss, Nucl. Phys B424 (1994) 308;

R. Kleiss, R. Pittau, Comp. Phys. Comm. 83 (1994) 141.

[18] L. Lönnblad et al., Comp. Phys. Comm. 70 (1992) 167.

[19] K. Hultqvist et al., Nucl. Instr. and Meth. A364 (1995) 193

[20] P. Billoir, Nucl. Instr. and Meth. A311 (1992) 139.

[21] ALEPH Collaboration, D. Buskulic et al., Phys. Reports 216 (1992) 253.

[22] DELPHI Collaboration, P. Abreu et al., CERN-PPE/96-03.

[23] L3 Collaboration, O. Adriani et al., Phys. Report 236 (1993) 1. 

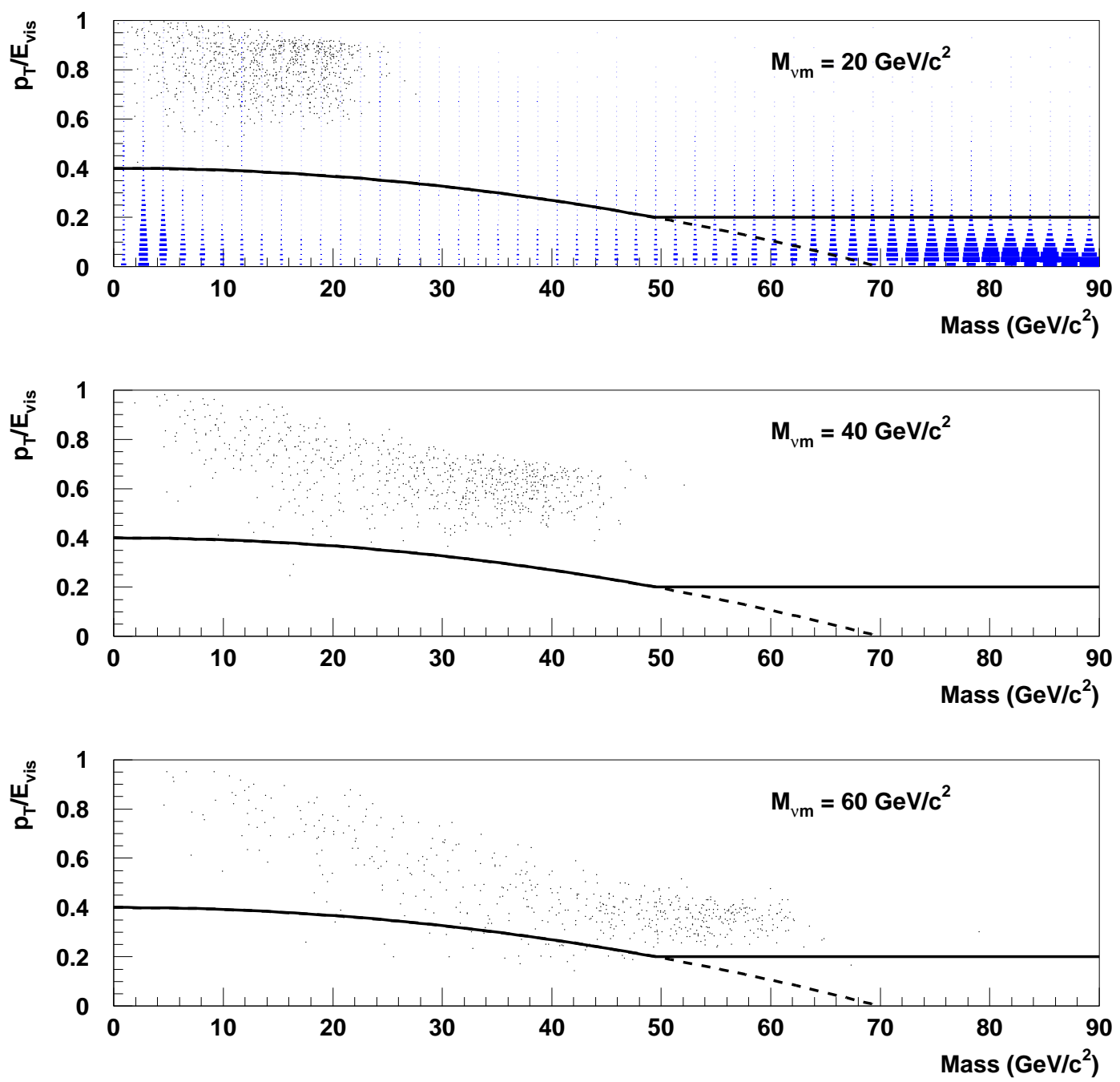

Figure 2: Dependence of the ratio $p_{t_{\mathrm{m} i s s}} / E_{\mathrm{v} \text { is }}$ on the invariant mass of the event for three different masses of the $\nu_{m}$. Only the events above the full line are accepted. The squares in the upper figure correspond to the real data while the points in all the plots correspond to the simulated Neutral Heavy Lepton signal. 


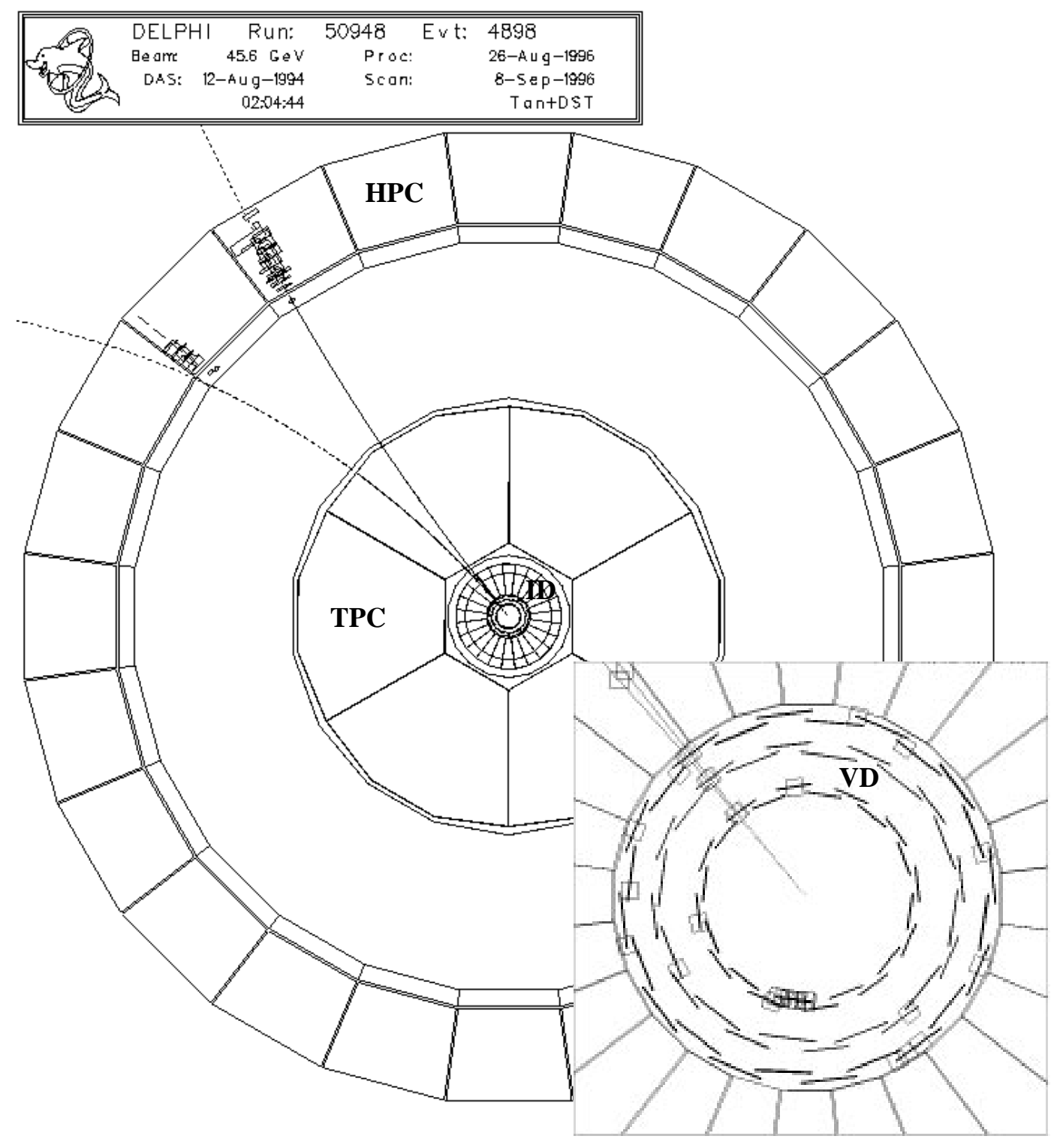

Figure 3: Surviving event in the monojet search. It has an invariant mass of $300 \mathrm{MeV} / \mathrm{c}^{2}$ and a missing $p_{t}$ of $6 \mathrm{GeV} / c$ and is probably an $e^{+} e^{-} \rightarrow e^{+} e^{-} \nu \bar{\nu}$ interaction. 


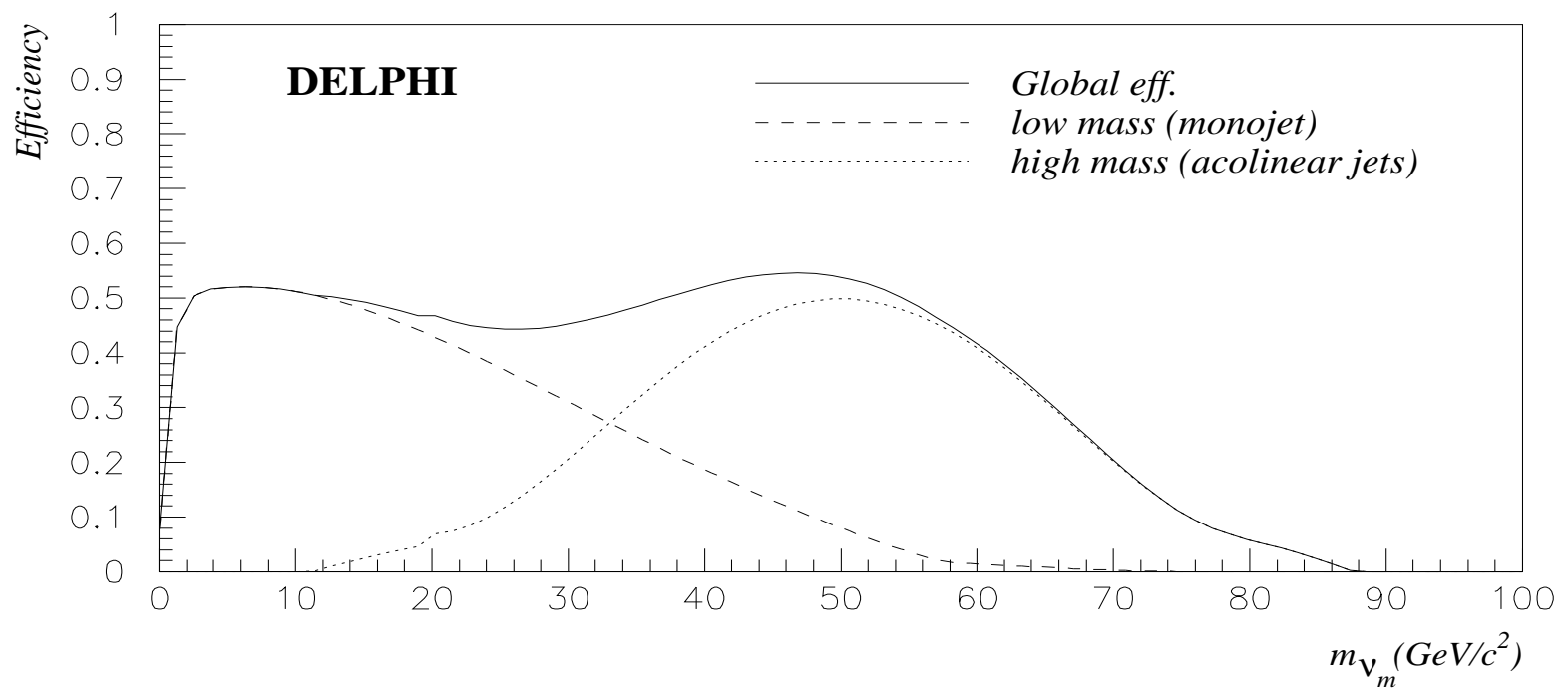

Figure 4: Efficiency of the monojet search (Section 3) and the acollinear jets search (Section 4). The full curve shows the efficiency of the two searches combined. 

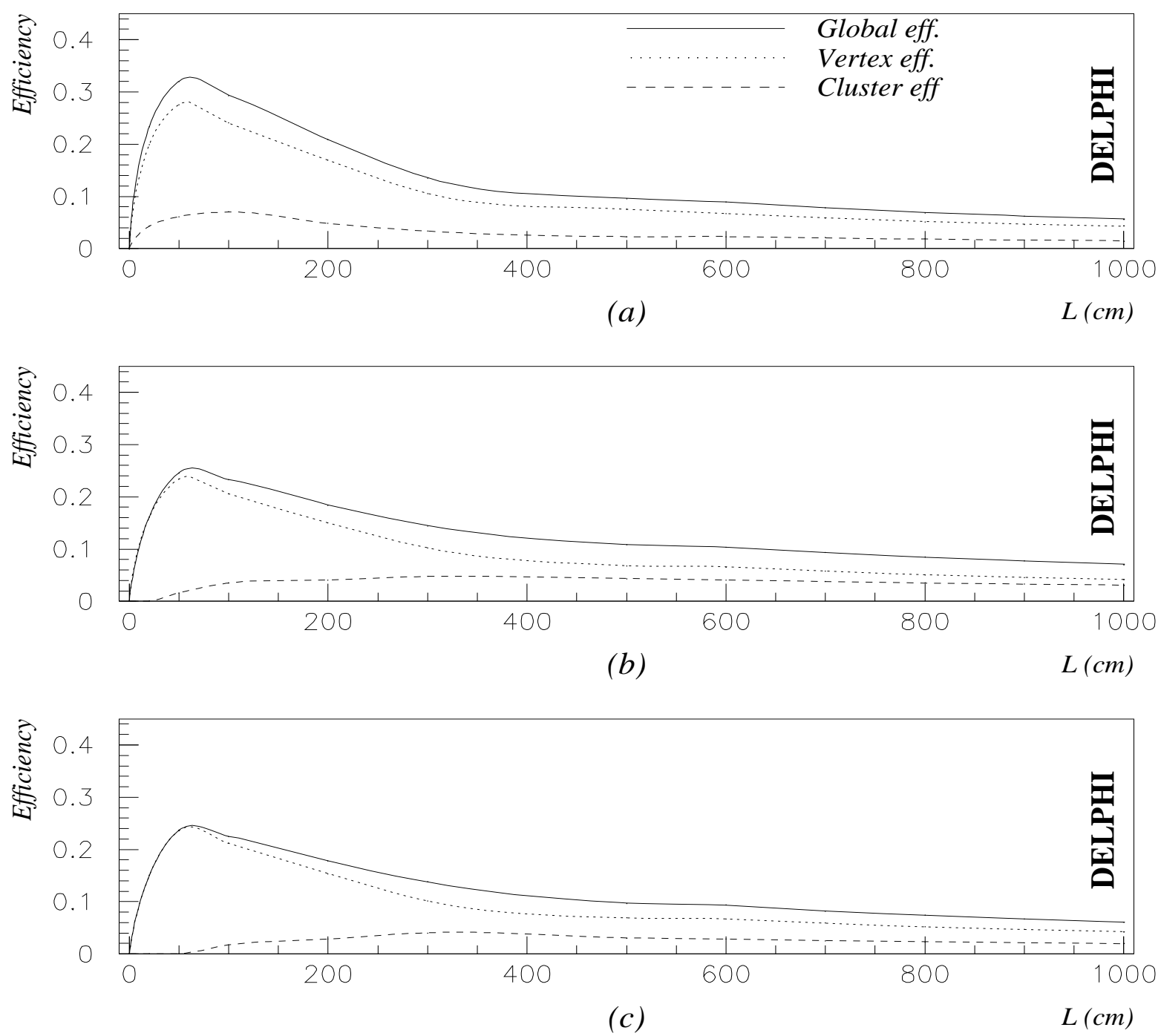

Figure 5: Efficiency of the vertex search (Section 5) and the cluster search (Section 6) for each $\nu_{m}$ decay channel, (a) $\nu_{m} \rightarrow \nu \ell \bar{\ell}$, (b) $\nu_{m} \rightarrow \nu \mathrm{q} \bar{q}$ and (c) $\nu_{m} \rightarrow \ell q \bar{q}^{\prime}$, as a function of the mean decay length $(L)$ for a fixed $\nu_{m}$ mass of $20 \mathrm{GeV} / c^{2}$. The full curves show the efficiency of the two searches combined. 

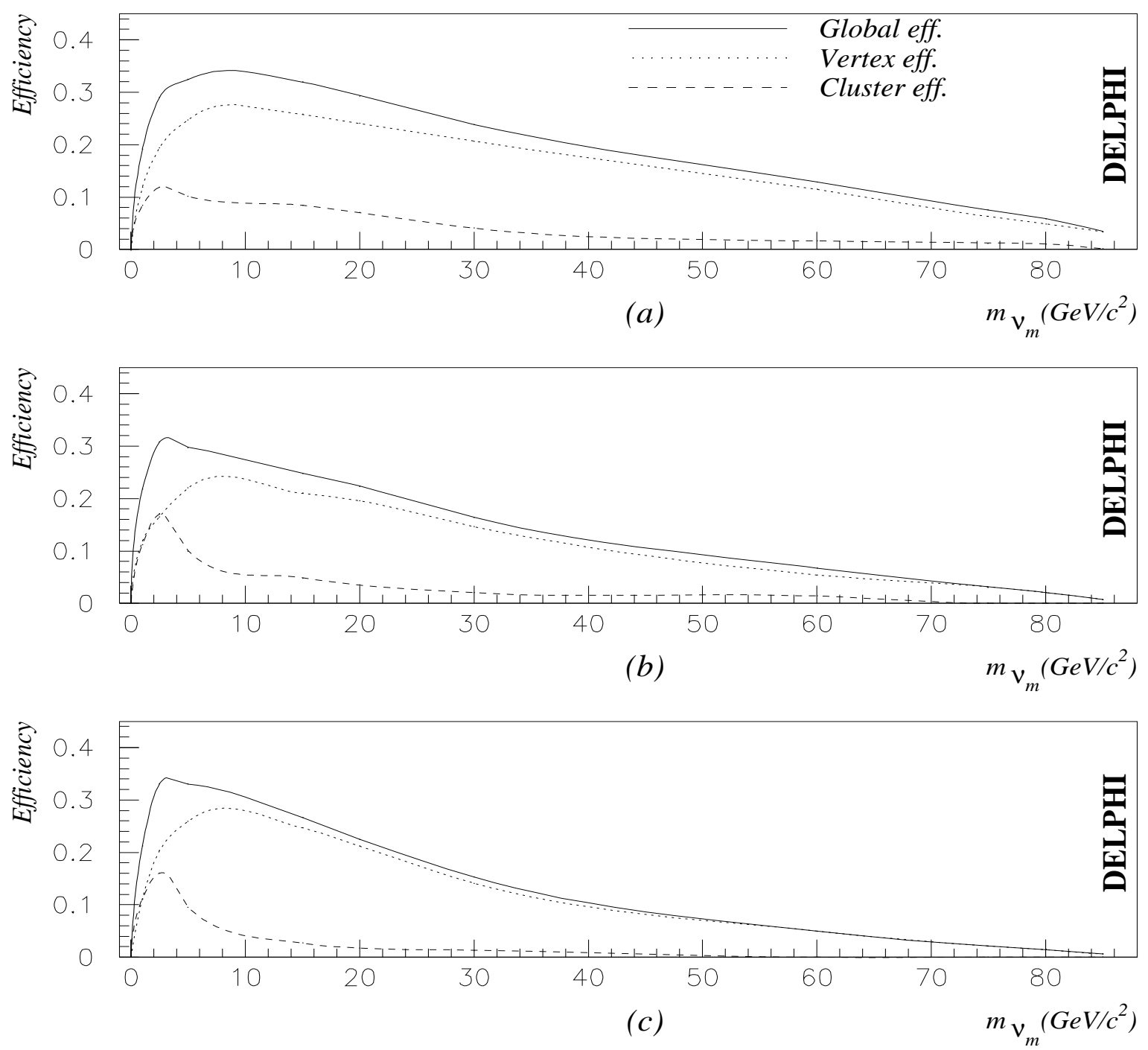

Figure 6: Efficiency of the vertex search (Section 5) and the cluster search (Section 6) for each $\nu_{m}$ decay channel, (a) $\nu_{m} \rightarrow \nu \ell \bar{\ell}$, (b) $\nu_{m} \rightarrow \nu q \bar{q}$ and (c) $\nu_{m} \rightarrow \ell q \bar{q}^{\prime}$, as a function of the $\nu_{m}$ mass for a fixed mean decay length $(L)$ of $100 \mathrm{~cm}$. The full curves show the efficiency of the two searches combined. 


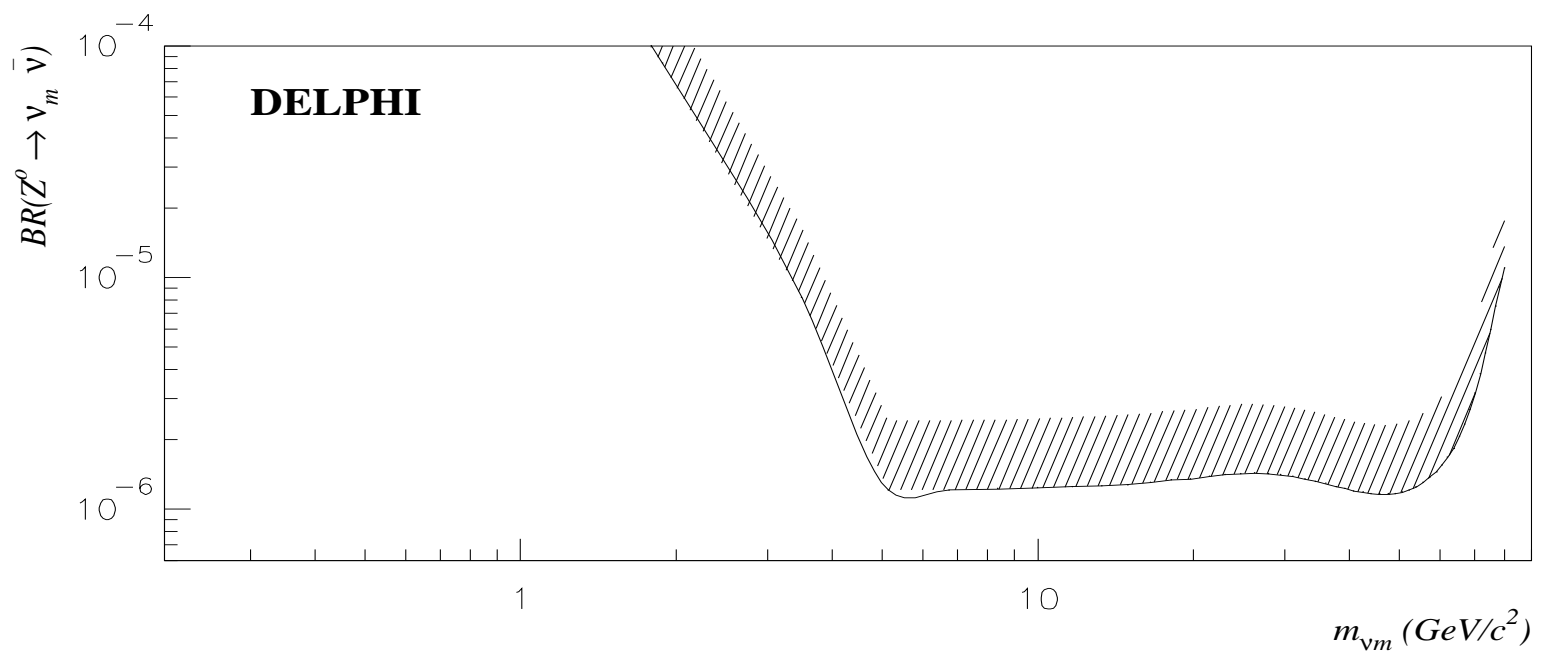

Figure 7: Limit at the $95 \% \mathrm{CL}$ on the branching ratio $B R\left(Z^{0} \rightarrow \nu_{m} \bar{\nu}\right)$ as a function of the $\nu_{m}$ mass. This limit has been obtained combining the monojet and acollinear jets searches for short-lived $\nu_{m}$ and taking into account Eq. 2 as explained in the text.
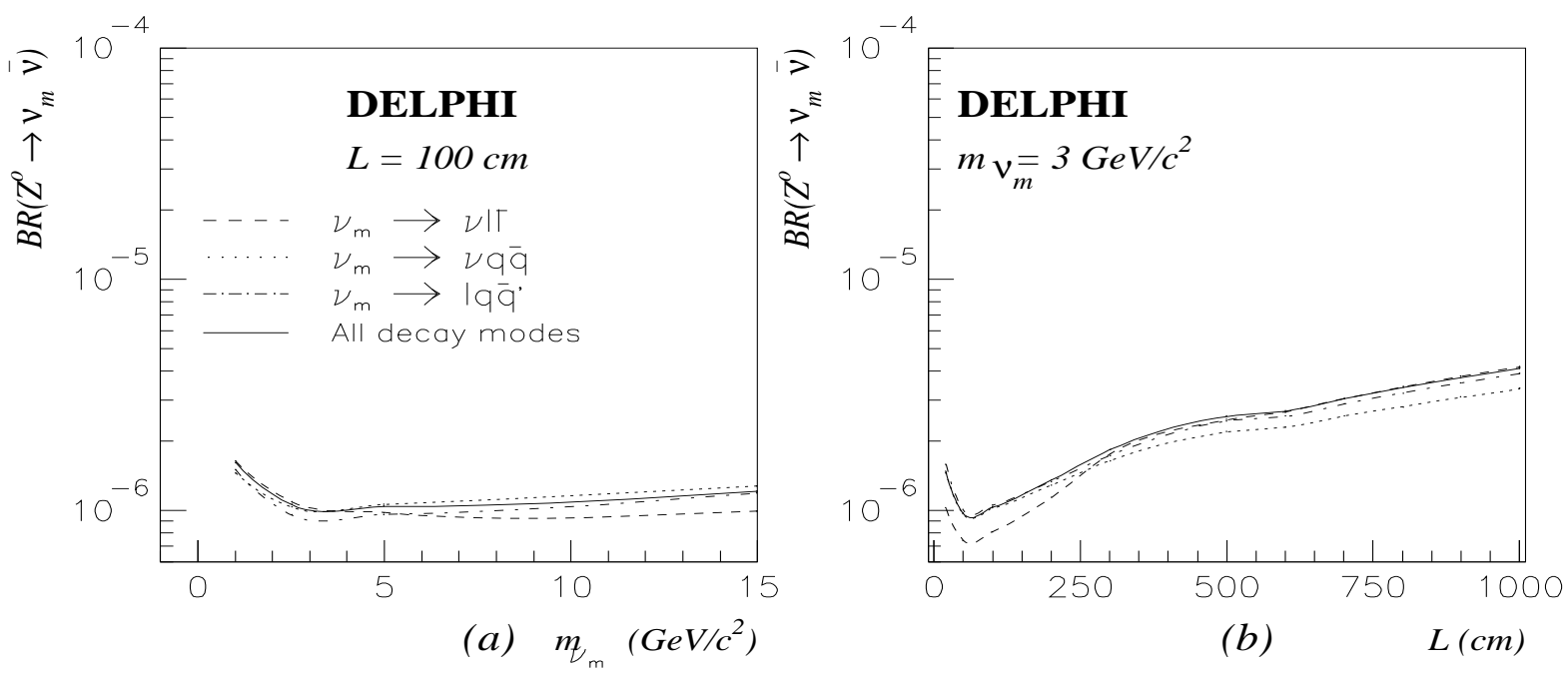

Figure 8: Limits at the $95 \% \mathrm{CL}$ for a long-lived $\nu_{m}$ on the branching ratio $B R\left(Z^{0} \rightarrow \nu_{m} \bar{\nu}\right)$ as a function of (a) the $\nu_{m}$ mass for a mean decay length $L$ of $100 \mathrm{~cm}$ and (b) the mean decay length $L$ for a $\nu_{m}$ mass of $3 \mathrm{GeV} / c^{2}$. These limits were obtained from the combined vertex and cluster searches firstly assuming for each decay mode a branching ratio of 1 (dashed, dotted and dash-dotted curves) and secondly combining all decay modes assuming $\operatorname{BR}\left(\nu_{m} \rightarrow \nu \ell \bar{\ell}\right)=0.24, \operatorname{BR}\left(\nu_{m} \rightarrow \nu q \bar{q}\right)=0.15$ and $\operatorname{BR}\left(\nu_{m} \rightarrow \ell q \bar{q}^{\prime}\right)=$ 0.55 (full curve), see section 1 . The limits shown here are not yet corrected according to Eq. 2. 

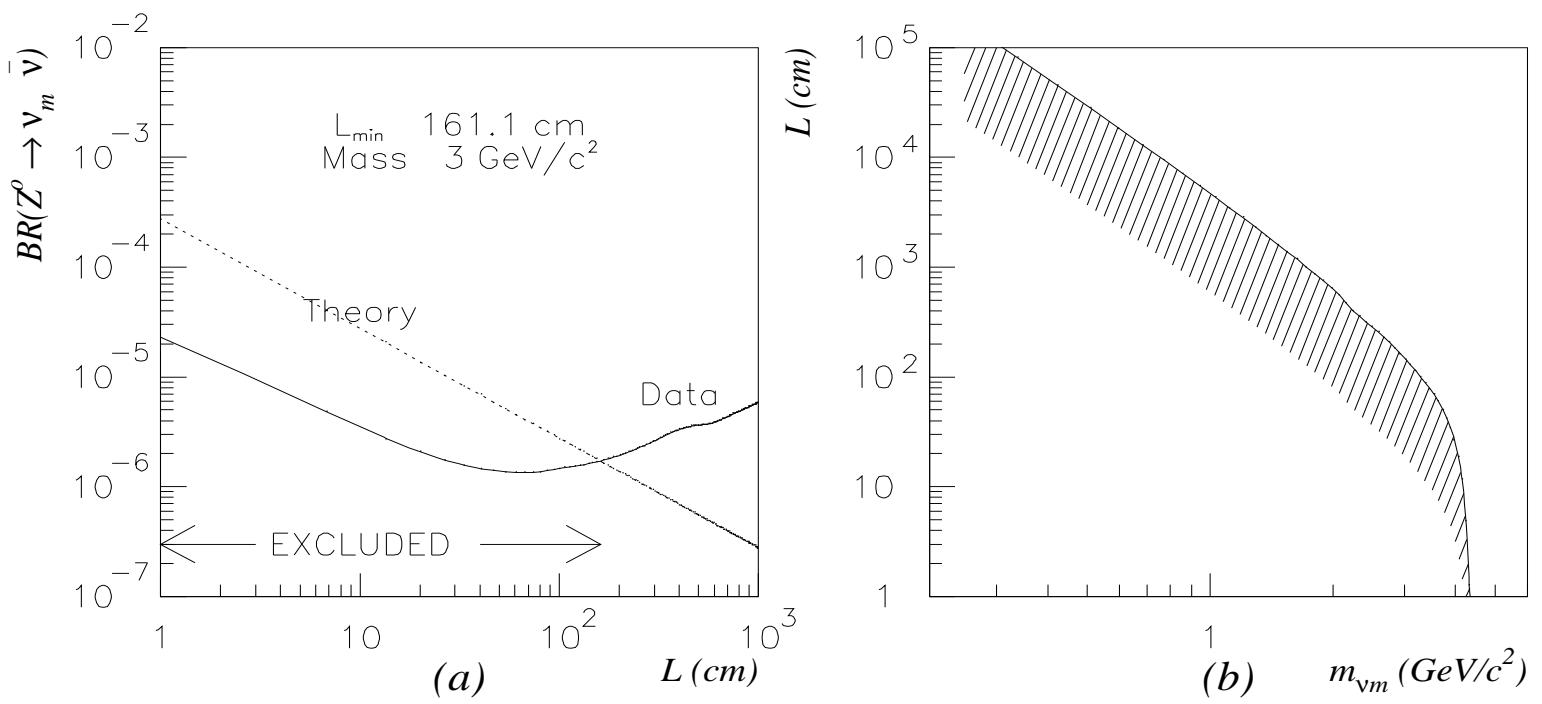

Figure 9: (a) Example of the method of determining the minimum allowed decay length $\left(L_{\text {min }}\right)$ for a $\nu_{m}$ with fixed mass $m_{\nu_{m}}$, here taken to be $3 \mathrm{GeV} / c^{2} . L_{\text {min }}$ is chosen as the minimum decay length for which the BR predicted by the theory (Eq. 1) is smaller than the 95\% CL upper limit allowed by the data for the given mass. (b) Region of excluded $\left(L, m_{\nu_{m}}\right)$ pairs is shown shadowed. The decay lengths on the exclusion curve are the $L_{m i n}$ values computed with the method sketched in (a).

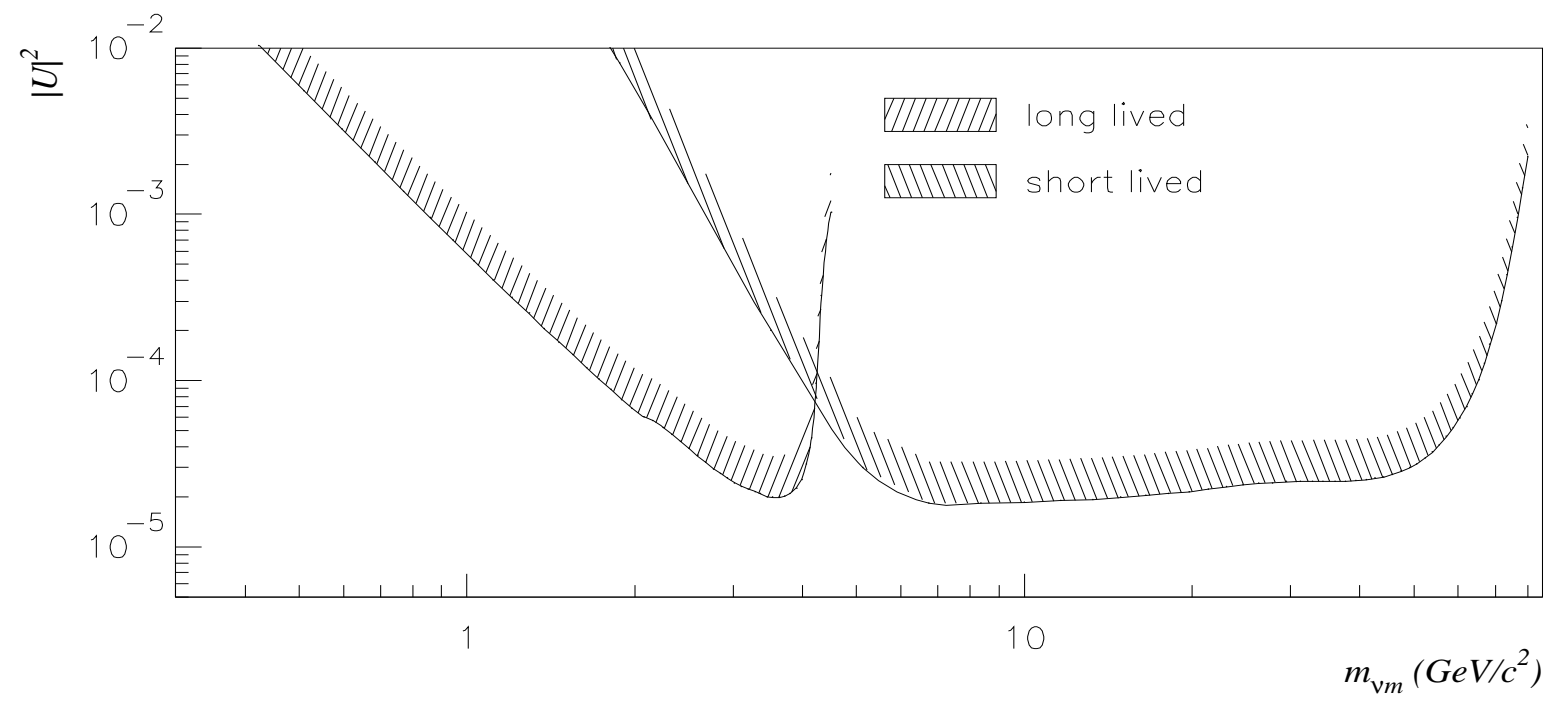

Figure 10: Limits at the $95 \% \mathrm{CL}$ for $|U|^{2}$ from the long and short-lived $\nu_{m}$ analyses as a function of the $\nu_{m}$ mass. The values have been corrected according to Eq. 2, as described in the text. 


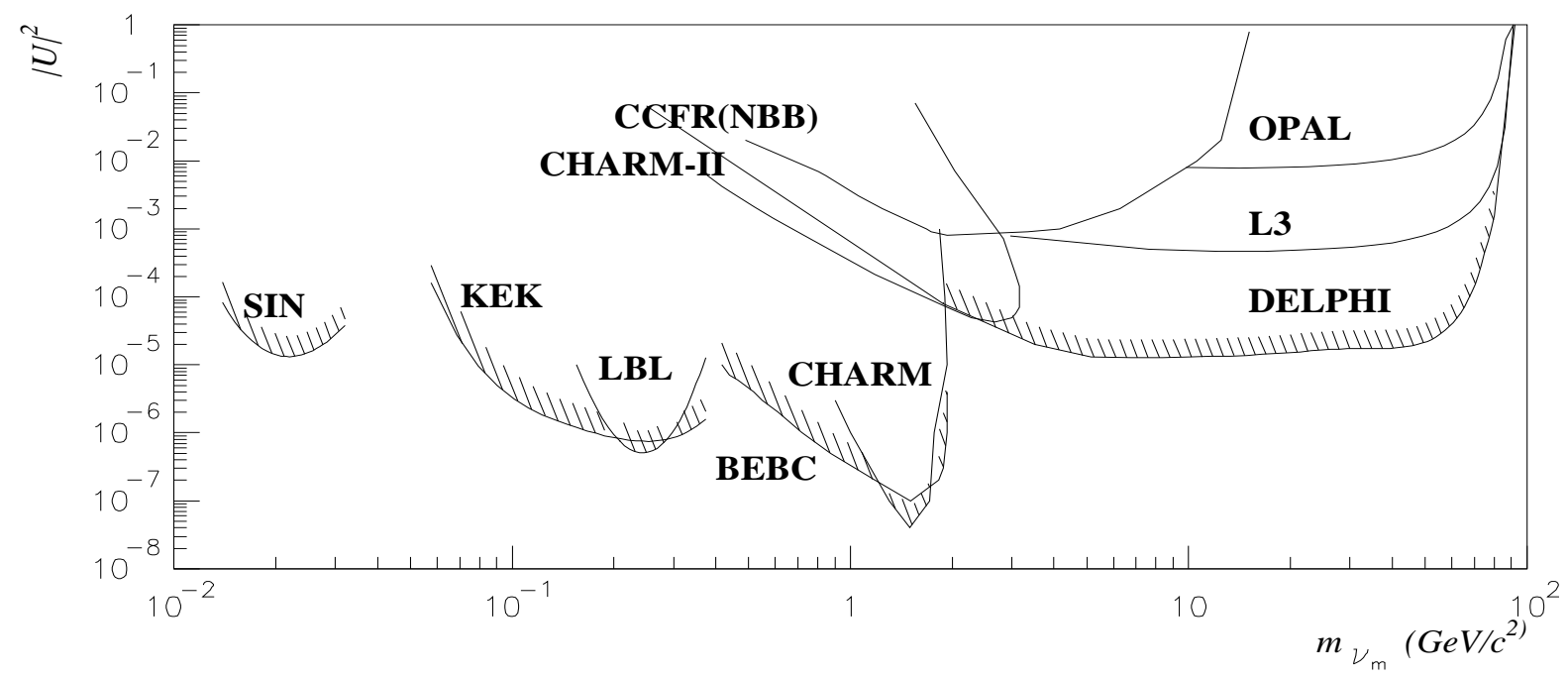

Figure 11: Limits at the $95 \% \mathrm{CL}$ on the mixing matrix element $|U|^{2}$ as a function of the $\nu_{m}$ mass for the various experiments referenced in the text. The limits shown for the present analysis correspond to those obtained combining the short-lived and long-lived $\nu_{m}$ analysis.

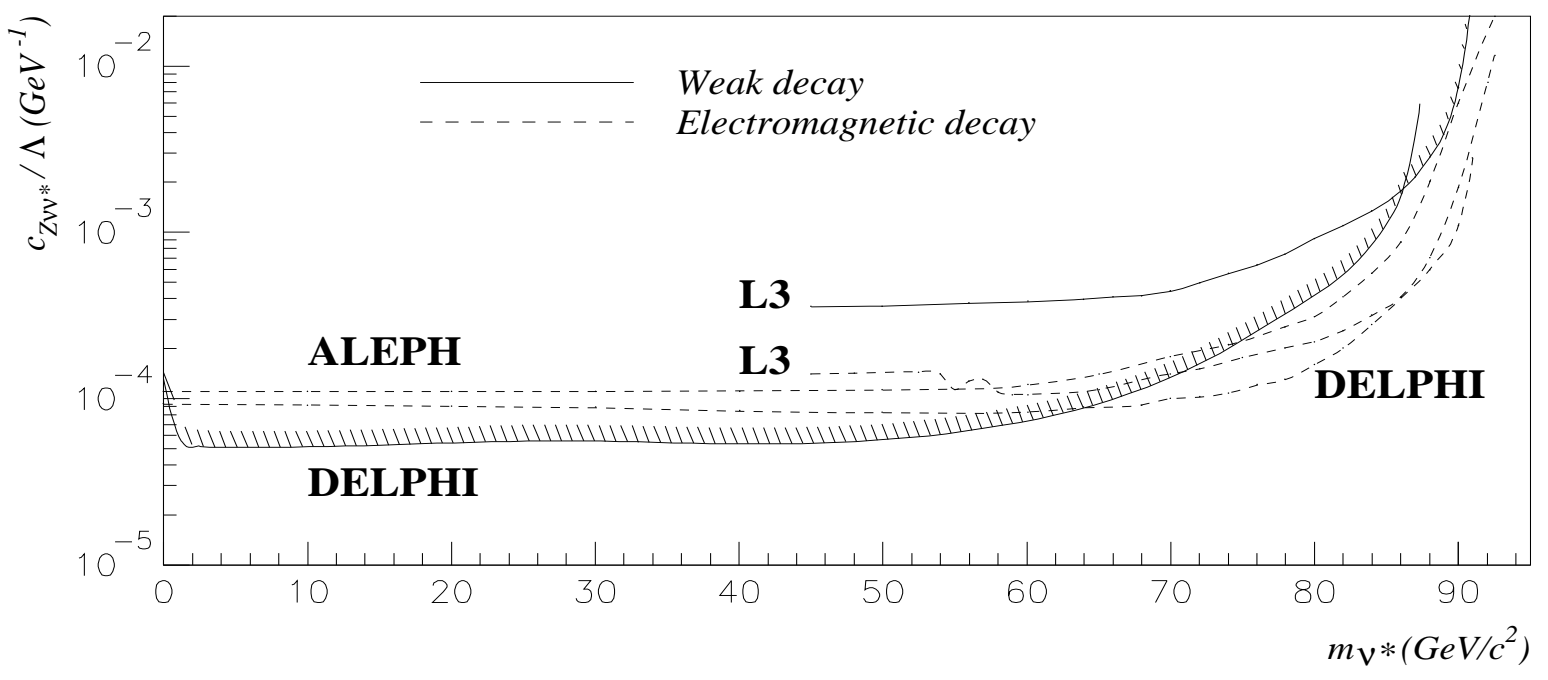

Figure 12: Upper limits at 95\% CL on the parameter $c_{Z^{0} \nu^{*} \nu} / \Lambda$ as a function of $m_{\nu^{*}}$ from the present analysis. For comparison, previous results from the LEP experiments referenced in the text are also shown. The full curves ('weak decay') correspond to the limits for the standard $\mathrm{SU}(2) \times \mathrm{U}(1)$ current, allowing only weak decays. The dashed curves ('electromagnetic decay') are the limits for $\nu^{*} \rightarrow \gamma \nu$, the dominant decay mode when the $\gamma \nu \nu^{*}$ coupling exists. 